\title{
Wall-resolved Large Eddy Simulation of turbulent mixed-convection heat transfer along a heated vertical flat plate
}

\author{
Ayodeji Ojofeitimi ${ }^{\mathrm{a}, *}$, Yasuo Hattori ${ }^{\mathrm{b}}$ \\ ${ }^{a}$ Department of Aerospace Engineering, University of Maryland-College Park, MD, 20740, USA \\ ${ }^{b}$ Fluid Science Sector, Central Research Institute of Electric Power Industry, 1646 Abiko, Abiko-shi, Chiba-ken, 270-1194, \\ Japan
}

\begin{abstract}
The present study aims to assess the predictive capabilities of wall-resolved large eddy simulation (LES) in computing the fluid flow and heat transfer characteristics in a mixed-convection turbulent boundary layer that developed along a large flat plate vertically mounted in air. The maximum Rayleigh number was approximately $3 \times 10^{11}$, which resulted in fully developed turbulence conditions. To enhance the accuracy, computational efficiency, and numerical stability, the LES solved the low-Mach number compressible flow governing equations, which included fluctuating density effects and pressure-density decoupling. For the subgrid scale $(S G S)$ closure, a locally dynamic Smagorinsky SGS model was implemented into the LES solver to enable the backscatter phenomenon intrinsic to transitioning boundary layer flows. The LES illustrated exceptional agreement with the statistics profiles in the boundary layer obtained with the experiments of previous studies, which showed sensitivity to freestream conditions. In particular, the LES correctly predicted the turbulent heat flux in the streamwise direction near the heated surface. Furthermore, the LES captured the rapid changes in spectra of fluctuating temperature and velocities due to the delay of transition with increasing freestream velocity.
\end{abstract}

Keywords: Turbulent boundary layer, Mixed convection, Turbulent heat transfer, Large Eddy Simulation, Boundary layer transition

\section{Introduction}

Many fluid transport processes encountered in nature and engineering applications are strongly affected by the presence of buoyancy. The buoyancy effect can be a consequence of temperature gradients within the flowfield. Such flowfield may be driven primarily by the buoyancy force (natural-convection) or it can be a combination of buoyancy and a weakly forced ambient flow induced via some mechanical means (mixedconvection). In these flows, the structural characteristics of the boundary layer are profoundly intricate, and it is a direct consequence of the non-linear effects and mutual coupling of the velocity and thermal flowfields.

Strongly affected buoyant flows are largely unstable and the flow mechanism can easily become turbulent. In mixed-convection boundary layer flows, the turbulent heat transfer characteristics not only depend upon the buoyancy induced temperature fluctuations, but also on the direction of the weakly forced ambient flow. The direction of the forced flow can be the same as the upward motion induced by buoyancy over a vertically heated flat plate (aiding flow) or in the opposite direction (opposite flow).

Due to the complexities intrinsic to mixed-convection turbulent boundary layer flows, there have been limited experimental and numerical studies of such flows over vertical flat surfaces. The turbulent characteristics of mixed-convection turbulent boundary layers in aiding flow have been experimentally studied to

\footnotetext{
* Corresponding author

Email address: aojofeitimi@outlook.com (A. Ojofeitimi). 


\begin{tabular}{|c|c|c|c|}
\hline \multicolumn{4}{|c|}{ Nomenclature } \\
\hline & & $\mathrm{x}, \mathrm{y}, \mathrm{z}$ & coordinate components, $\mathrm{m}$ \\
\hline I & unit tensor & \multicolumn{2}{|c|}{ Greek symbols } \\
\hline $\mathbf{L}$ & Leonard stress tensor & $\alpha$ & thermal diffusivity, $\mathrm{m}^{2} / \mathrm{s}$ \\
\hline $\mathbf{M}$ & rate of strain tensor & $\beta$ & volume expansion coefficient, $1 / \mathrm{K}$ \\
\hline $\mathbf{S}$ & rate of strain tensor & $\Delta$ & difference operator \\
\hline $\begin{array}{l}\mathrm{u} \\
\mathrm{x}\end{array}$ & $\begin{array}{l}\text { velocity instantaneous, } \mathrm{m} / \mathrm{s} \\
\text { general spatial coordinate, } \mathrm{m}\end{array}$ & $\delta$ & $\begin{array}{l}\text { momentum boundary layer integral } \\
\text { length, } \mathrm{m}\end{array}$ \\
\hline$C_{d}$ & dynamic coefficient & $\infty$ & freestream/ambient condition \\
\hline$c_{p}$ & $\begin{array}{l}\text { specific heat at constant pressure, } \mathrm{kJ} /(\mathrm{kg} \\
\mathrm{K})\end{array}$ & $\begin{array}{l}\kappa \\
\mu\end{array}$ & $\begin{array}{l}\text { thermal conductivity, } \mathrm{W} /(\mathrm{m} \mathrm{K}) \\
\text { viscosity, } \mathrm{Pa} \mathrm{s}\end{array}$ \\
\hline$G r_{x}$ & local Grashof number, $\frac{g \beta\left(T_{w}-T_{\infty}\right) x^{3}}{\nu^{2}}$ & $\nabla$ & gradient operator \\
\hline$R e_{x}$ & local Reynolds number, $U_{\infty} x / \nu$ & $\nu$ & kinematic viscosity, $\mathrm{m}^{2} / \mathrm{s}$ \\
\hline$R i_{x}$ & local Richardson number, $G r_{x} / R e_{x}^{2}$ & $\rho$ & density, $\mathrm{kg} / \mathrm{m}^{3}$ \\
\hline$t^{\prime}$ & temperature fluctuation, $\mathrm{K}$ & $\sigma$ & stress, $\mathrm{Pa}$ \\
\hline$u^{\prime}, v^{\prime}$ & fluctuating velocity components, m/s & $\tau$ & shear stress, $\mathrm{Pa}$ \\
\hline$u^{\prime} t^{\prime}$ & streamwise turbulent heat flux, $(\mathrm{m} \mathrm{K}) / \mathrm{s}$ & \multicolumn{2}{|c|}{ Oversymbols } \\
\hline$u^{\prime} v^{\prime}$ & Reynolds Shear Stress, $m^{2} / s^{2}$ & - & ensemble-averaged quantity \\
\hline$y^{*}$ & non-dimensional wall unit & $\sim$ & Favre filtered \\
\hline $\mathrm{g}$ & gravitational acceleration, $\mathrm{m} / \mathrm{s}^{2}$ & - & test filter \\
\hline h & enthalpy, J & \multicolumn{2}{|c|}{ Subscripts } \\
\hline p & pressure, $\mathrm{Pa}$ & \multirow{2}{*}{\multicolumn{2}{|c|}{$\begin{array}{l}B 1, B 2, B 3 \text { streamwise direction mesh related } \\
\text { scales }\end{array}$}} \\
\hline $\operatorname{Pr}$ & Prandtl, $\mu c_{p} / \kappa$ & & \\
\hline $\mathbf{q}, \mathbf{q}$ & heat flux, $W / m^{2}$ & $S G S$ & sub-grid scale \\
\hline $\mathrm{R}$ & universal gas constant, $\mathrm{J} /(\mathrm{K} \mathrm{mol})$ & $V S L$ & viscous sublayer \\
\hline $\mathrm{T}$ & temperature, $\mathrm{K}$ & & wall \\
\hline $\mathrm{t}$ & time, $\mathrm{s}$ & \multicolumn{2}{|c|}{ Superscripts } \\
\hline $\mathrm{U}$ & mean streamwise velocity, $\mathrm{m} / \mathrm{s}$ & 1 & fluctuating component \\
\hline W & molecular weight of air, $\mathrm{kg} / \mathrm{kmol}$ & $\prime \prime$ & deviatoric component \\
\hline
\end{tabular}

elucidate the fundamental structure of the boundary layer [11-14, 21, 22]. These measurements indicated the decrease in wall heat transfer was attributable to suppression of turbulence in the mixed-convection turbulent boundary layer. Several researchers have similarly attempted to numerically investigate mixed-convection turbulent wall flows over heated vertical flat surfaces $[2,20,31]$. Direct numerical simulation (DNS) [2, 20] and Reynolds Averaged Navier Stokes (RANS) [31] results illustrated that the Nusselt number decreased as the freestream velocity was imposed on the natural-convection turbulent boundary layer. Furthermore, the DNS results revealed the turbulent heat transfer in aiding and opposite flows differs significantly [21]. To date, there is no detailed LES-based computational study of mixed-convection turbulent wall flows over 
heated vertical flat surfaces. This can be attributed to the fact that the simulation of mixed-convection turbulent boundary layers remains a formidable task. One of the difficulties entailed in computing buoyancy affected wall flows is that the classical analysis developed for momentum-driven turbulent wall flows based upon the decomposition of the near-wall region into a viscous sublayer and logarithmic layer needs to be carefully adapted. A second difficulty is that such flows often correspond to high Reynolds numbers (i.e. high Grashof and Rayleigh numbers), and it can be computationally expensive and numerically challenging to resolve the turbulent heat transfer near the wall since momentum driven turbulent flow classical inner layer scalings do not strictly apply. These numerical challenges were observed in the LES computations of [3, 27] and ergodicity-based DNS of [1,2], all of which employed artificial treatments to reproduce the transition from laminar to turbulence in the boundary layer, have not firmly grasped the turbulence characteristics in the near-wall region.

The utilization of large eddy simulation in computing turbulent boundary layers follows two approaches [32], namely fine-grained and coarse grained computations. Fine-grained simulations correspond to wall-resolved simulations, whereby the viscous sublayer is fully resolved to capture the near-wall small scale turbulence physics, and the first off-the wall grid node is within the viscous sublayer. However, the constraint associated with performing fine-grained LES calculations lies in the fact that at high Reynolds numbers, i.e. thin viscous sublayer, the computational requirements becomes prohibitively expensive for engineering-level simulations. Coarse-grained simulations correspond to wall-layer modeled simulations where approximate equations are utilized to estimate the near-wall viscous sublayer region. The approximate equations are used to reconstruct the wall-shear stress and wall-heat flux, and the first off-the wall grid node is usually outside the viscous sublayer.

In the present study, we present wall-resolved large eddy simulation of mixed-convection turbulent boundary layer flows over an isothermal heated vertical flat plate configuration and address the shortcomings of previously mentioned works, i.e. their inability to accurately resolve the laminar to turbulence transition "naturally" and near-wall turbulent flow characteristics. The canonical configuration corresponds to experimental measurements previously performed by Hattori et al. [12-14], which dealt with the rapid change in the transition process and near-wall structures of a natural-convection boundary layer as the freestream velocity is increased.

\section{Approach}

\subsection{Experimental configuration}

As described in Refs. [13, 14], the experimental configuration corresponds to an isothermal heated flat plate inside a low-speed vertical wind tunnel. The wind tunnel comprises a blower which can force air into the test section up to $10 \mathrm{~m} / \mathrm{s}$. Downstream of the blower resides a diffuser with a relatively large cone angle that can slow down the flow enough to induce flow separation. Thus, three safety screens were positioned in the diffuser to smooth out variations in the velocity field. To dampen freestream disturbance or turbulence, four fine mesh damping screens along with a honeycomb were placed in the settling chamber.

The dimensions of the plate were $4 \mathrm{~m}$ high and $0.8 \mathrm{~m}$ wide to generate two-dimensional turbulent flow, and $0.02 \mathrm{~m}$ thick to prevent deformation due to thermal stress. The plate was mounted vertically on the back wall of the vertical wind tunnel. The temperature of the plate was kept uniform at approximately 370 Kelvin via electric heaters implemented at the rear of the plate. The ambient temperature for the experiments was approximately 300 Kelvin. Temperature and velocity data in the turbulent boundary layer were measured with tungsten hot wire and cold wire using a sampling frequency of $500 \mathrm{~Hz}$. Table 1 gives the experimental test conditions that will be numerically investigated with wall-resolved LES. The three experimental test conditions were distinguished using the non-dimensional local Richardson number, $R i_{x}=G r_{x} / R e_{x}^{2}$, which is a measure of buoyancy to inertial effects.

\subsection{Numerical solver}

The numerical solver employed in the present study was lowMachFOAM [28]. The code is based upon OpenFOAM $^{\circledR}$, which is a free open-source CFD software package produced by OpenCFD Ltd. [30]. lowMachFOAM is an object-oriented, $\mathrm{C}++$ based, second-order accurate, finite volume solver with implicit 


\begin{tabular}{|l|c|c|c|}
\hline Case & $U_{\infty}(\mathrm{m} / \mathrm{s})$ & $T_{\infty}$ (Kelvin) & $T_{w}$ (Kelvin) \\
\hline \hline$R i_{x}=\infty$ & 0.00 & $\sim 300$ & $\sim 370$ \\
\hline$R i_{x}=39.42$ & 0.53 & $\sim 300$ & $\sim 370$ \\
\hline$R i_{x}=15.27$ & 0.80 & $\sim 300$ & $\sim 370$ \\
\hline
\end{tabular}

Table 1: Experimental test conditions.

time integration. The solver has advanced built-in mesh generation capabilities, which allows for either structured or unstructured polyhedral mesh geometries. In addition, the solver features a massively parallel computing capability utilizing message passing interface (MPI) protocols.

lowMachFOAM utilizes a Favre-filtered low-Mach number formulation as opposed to solving the full compressible flow governing equations. The purposes of the low-Mach number assumptions are twofold: (1) eliminate compressibility effects by filtering acoustic waves. Consequently, the numerical calculation will be affected and dictated only by speeds comparable to the flow velocity as opposed to the sound speed, and (2) the modified equations will consist of fewer terms, thereby reducing the computational effort during the numerical simulations. Using the proposed assumptions, the Favre-filtered differential equations consisting of the mass, momentum, and energy conservation equations, respectively are:

$$
\begin{gathered}
\frac{\partial \bar{\rho}}{\partial t}+\nabla \cdot \bar{\rho} \tilde{\mathbf{u}}=0 \\
\frac{\partial \bar{\rho} \tilde{\mathbf{u}}}{\partial t}+\nabla \cdot \bar{\rho} \tilde{\mathbf{u}} \tilde{\mathbf{u}}-\nabla \cdot \tilde{\sigma}=-\nabla \bar{p}+\bar{\rho} \mathbf{g}-\nabla \cdot \tau_{S G S} \\
\frac{\partial \bar{\rho} \tilde{h}}{\partial t}+\nabla \cdot \bar{\rho} \tilde{h} \tilde{\mathbf{u}}-\frac{\partial \bar{p}}{\partial t}-\tilde{\mathbf{u}} \cdot \nabla \bar{p}+\nabla \cdot \tilde{\mathbf{q}}=-\nabla \cdot \mathbf{q}_{S G S}
\end{gathered}
$$

The majority of subgrid scale stress tensor, $\tau_{S G S}$, models are based on the eddy viscosity concept, wherein the subgrid scale stress is expressed linearly in terms of the resolved velocity gradient and coefficients that depend on some defined flow characteristics [34]. The $S G S$ stress tensor is modeled with the following mathematical expression:

$$
\tau_{S G S}-\frac{1}{3} \operatorname{tr}\left(\tau_{S G S}\right) \mathbf{I}=-2 C_{d} \Delta^{2} \bar{\rho}|\tilde{\mathbf{S}}|\left(\tilde{\mathbf{S}}-\frac{1}{3} \operatorname{tr}(\tilde{\mathbf{S}}) \mathbf{I}\right)
$$

The isotropic part of the subgrid scale stress tensor, $\operatorname{tr}\left(\tau_{S G S}\right) \mathbf{I}$, has been shown to be insignificant for low-speed turbulent boundary layers [7]. Moreover, the deviatoric part of the $S G S$ stress tensor, $\tau_{S G S}$, employs the eddy viscosity concept by assuming that it is locally aligned with the deviatoric part of the resolved strained rate tensor [8]. To estimate the subgrid-scale tensor field, we employ the locally dynamic Smagorinsky $S G S$ model. The coefficient $C_{d}$ is determined dynamically, rather than input a priori, and reads as

$$
C_{d}=-\frac{1}{2} \frac{\langle\mathbf{L}: \mathbf{M}\rangle}{\langle\mathbf{M}: \mathbf{M}\rangle},
$$

where the $\langle$.$\rangle denotes some ensemble average. The ensemble averaging procedure is required to avoid$ excessively large fluctuations of the coefficient [23] that can otherwise destabilize the numerical simulation.

To formally describe the dynamic procedure, a second, coarser spatial filter or test filter is applied to the filtered equations of motions. The test filter width is usually defined as $\hat{\Delta}=2 \Delta$; the caret, ${ }^{\wedge}$, will henceforth denote the test filter. The Leonard stress, $\mathbf{L}$, can be mathematically stated using the Germano identity [10] and adopting the approach of [26]:

$$
\mathbf{L}=\widehat{\bar{\rho} \tilde{\mathbf{u}} \tilde{\mathbf{u}}}-\frac{\widehat{\bar{\rho} \tilde{\mathbf{u}}} \widehat{\bar{\rho} \tilde{\mathbf{u}}}}{\widehat{\bar{\rho}}}
$$


The Leonard stress defined for the dynamic procedure is basically the contribution to the Reynolds stresses by turbulence scales intermediate between the grid filter and test filter scales. The tensor quantity $\mathbf{M}$ is defined as

$$
\mathbf{M}=\eta-\hat{\zeta},
$$

where $\zeta$ and $\eta$ are the resolved fields at the subgrid scale and subtest filter scale, respectively:

$$
\begin{gathered}
\zeta=\Delta^{2} \bar{\rho}|\tilde{\mathbf{S}}|\left(\tilde{\mathbf{S}}-\frac{1}{3} \operatorname{tr}(\tilde{\mathbf{S}}) \mathbf{I}\right) \\
\eta=\widehat{\Delta^{2}} \hat{\bar{\rho}}|\widehat{\tilde{\mathbf{S}}}|\left(\widehat{\tilde{\mathbf{S}}}-\frac{1}{3} \operatorname{tr}(\widehat{\tilde{\mathbf{S}}}) \mathbf{I}\right) .
\end{gathered}
$$

It is critical to note that subsequent to the computation of the dynamic coefficient $C_{d}$, this quantity was locally averaged to smooth out potentially large fluctuations. Following the local averaging procedure, backscatter was enabled by allowing the SGS viscosity to acquire a minimum value of $-\nu$. Since all the flows considered in the present study are transition to turbulent wall bounded flows, it was imperative to capture the backscatter inherent to transitioning boundary layers [33].

The approach normally taken to modeling the subgrid scale heat flux, $\mathbf{q}_{S G S}$, is based on the premise that the energy transfer from resolved to unresolved turbulence scales is proportional to the resolved temperature, $\tilde{T}$ [24], and it is modeled as

$$
\mathbf{q}_{S G S}=-\bar{\rho} \alpha_{S G S} \nabla \tilde{h},
$$

where $\alpha_{S G S}$ is the subgrid scale thermal diffusion coefficient and can be defined as

$$
\alpha_{S G S}=\frac{\nu_{S G S}}{P r_{S G S}}
$$

$\nu_{S G S}$ and $\operatorname{Pr}_{S G S}$ are the subgrid scale kinematic viscosity and Prandtl number, respectively. The range of values chosen for a fixed $\operatorname{Pr}_{S G S}$ is $[0.3,0.9]$ [9]. The present study utilized a fixed value of 0.9 .

Before we proceed, a discussion is warranted on the treatment of the pressure term, $\bar{p}$, in Eqs. (2) and (3). Let us consider decomposing the pressure term by employing a similar procedure proposed by [6]; the pressure is split into a reference or ambient pressure, hydrostatic pressure, and a modified dynamic pressure respectively, as follows:

$$
\bar{p}=p_{\text {ref }}+\bar{\rho} \mathbf{g} \cdot \mathbf{x}+\bar{p}_{d} .
$$

Since the assumption that the coupling between pressure and density is non-existent, we can assume that temperature and density are inversely proportional in low-Mach number flows [25]. To be more specific, the pressure-density decoupling assumes the density is not dependent upon the modified pressure or dynamic pressure term, $\bar{p}_{d}$, since it is linked to the speed of the flow. This means that density is a function of the thermodynamic pressure, $\bar{p}_{t h}$. Thus, we can define the equation of state as follows for low-Mach number flows:

$$
\bar{p}_{t h}=\frac{\bar{\rho} R \tilde{T}}{W},
$$

where $\bar{p}_{t h}=p_{\text {ref }}+\bar{\rho}$ g.x. Utilizing the pressure decomposition methodology, the filtered energy equation, Eq.(3), can be simplified by substituting $\bar{p}_{t h}$ for the pressure term, $\bar{p}$. For the filtered momentum equation, Eq. (2), decomposing the pressure term results in a need to estimate the $\overline{p_{d}}$ field. Hence, we employ the identical methodology of $[18,19]$ to derive a similar modified dynamic pressure equation with the inclusion of density gradients due to wall heat transfer. The $\overline{p_{d}}$ equation was derived by substituting the semi-discretized momentum equation for $\tilde{\mathbf{u}}$ into the continuity equation. Proceeding in this manner results in the following semi-discrete expression: 


$$
\nabla \cdot\left(\bar{\rho} \mathbf{a}_{\mathbf{P}}{ }^{-1} \nabla \bar{p}_{d}\right)=\nabla \cdot\left(\bar{\rho} \mathbf{a}_{\mathbf{P}}{ }^{-1} \mathbf{H}(\tilde{\mathbf{u}})\right)-\nabla \cdot\left(\bar{\rho} \mathbf{a}_{\mathbf{P}}{ }^{-1} \mathbf{g} \cdot \mathbf{x} \nabla \bar{\rho}\right)+\frac{\partial \bar{\rho}}{\partial t},
$$

where the matrix coefficients $\mathbf{a}_{\mathbf{P}}{ }^{-1}$ and $\mathbf{H}(\tilde{\mathbf{u}})$ are representative of diagonal and off-diagonal parts of the solution matrix that emerged from the finite volume discretization. Due to the fact that the flows treated in the current work are not convection dominated, a second-order spatial central differencing scheme was employed to discretize the convection terms. The diffusion terms were discretized using the same approach discussed in [28]. The second-order Crank-Nicholson implicit time integration scheme was used to advance the solution in time, wherein the simulations were initialized with a time-step of $\Delta t=10^{-4} \mathrm{~s}$. The timestep was adjusted accordingly by the evolving flowfield as the solution process progressed using the Courant number (CFL) criterion, $\frac{\tilde{u} \Delta t}{\Delta x}+\frac{\tilde{v} \Delta t}{\Delta y}+\frac{\tilde{w} \Delta t}{\Delta z} \leqslant$ CFL. The Courant number was fixed to a value of 0.5 for the entire duration of the numerical simulations. The Pressure-Implicit with Splitting of Operators (PISO) algorithm was utilized for the pressure-velocity calculation procedure in which the discretized momentum and energy equations were implicitly predicted. The modified dynamic pressure equation, Eq.14, is then solved and used to correct the momentum equation (velocity field) to satisfy conservation of mass. Elaborate discussions on the PISO algorithm can be found in [16, 17, 29].

\subsection{Numerical configuration}

The computational domain and boundary conditions are graphically represented in Fig. 1. Uniform freestream values, $U_{\infty}$ and $T_{\infty}$, were imposed at the inflow to match the experimental values. Downstream of the inflow region, a $0.1 \mathrm{~m}$ long entrance domain was implemented to prevent an abrupt start of the boundary layer. The length of the flat plate was chosen to be $5 \mathrm{~m}$ to disallow potentially corrupted solution that can develop near the outflow to affect the region of interest.

At the outflow, the boundary condition was such that if the velocity vector normal to the boundary pointed out of the computational domain, a Von-Neumann numerical boundary is applied. However, if the normal velocity vector is into the computational domain, i.e. backflow, the numerical solution recomputes the outflow region with a Dirichlet numerical boundary by setting the velocity to zero and other flowfield quantities to ambient values. On the solid boundary, the boundary conditions have to be properly specified in accordance with the actual boundaries that exist in the flow, such as the no-slip impermeable and isothermal wall conditions. In addition, a wall-normal pressure boundary condition that assumes $\frac{\partial \bar{p}_{d}}{\partial n}=-\frac{\partial \bar{\rho}}{\partial n} \mathbf{g}$. $\mathbf{x}$ was imposed. This particular boundary condition is conducive to higher fidelity calculations and increased numerical stability by including the strongly buoyant flow condition at the wall. An identical numerical boundary equation for $\bar{p}_{d}$ was utilized at the inflow and outflow computational boundaries. The far-field boundary, which spanned $2 \mathrm{~m}$ in the wall-normal direction, was implemented to be sufficiently far away from the wall region because the spatially growing turbulent boundary layer will accelerate the prescribed non-zero freestream velocity, and its effects can be mitigated by positioning the boundary far from the wall. The entrainment boundary imposed at the far-field can either be an inflow or outflow boundary as the simulation progresses. Thus, during the solution process, if the flux is into the domain, the velocity normal to the computational boundary is calculated using a total pressure boundary condition with a specified inflow pressure. However, if the flux is out of the domain, the velocity gradient normal to the boundary is set to zero.

Periodic boundaries were implemented in the spanwise direction. The spanwise direction width of the computational domain was approximated to be $3 \delta$. The integral length scale, $\delta$, was taken to be the wallnormal length from the hot wall to the region where the mean streamwise velocity "reached" the prescribed freestream value. The approximate value of $\delta$ was acquired from the experimental data of [12]. For example, from Fig.4a, the approximate integral length scale for the $R i_{x}=\infty$ flow configuration was $\delta \approx 0.30 \mathrm{~m}$.

To capture the intricate small-scale physics inherent to laminar-turbulent transition, the streamwise direction grid resolution, $\Delta x$, was uniform in the laminar-transition region. Uniform grid was required to capture the formation and evolution of turbulent spots in the laminar-transition region. Once the flow acquired a fully developed turbulent state, the grid resolution was coarsened and subsequently kept constant in the streamwise direction. This resulted in three mesh blocks in the streamwise direction. Uniform grid 


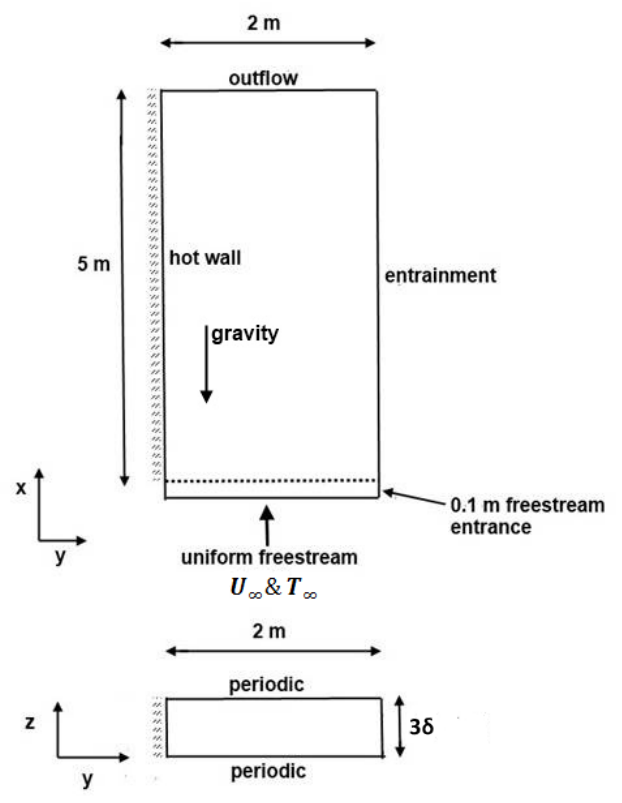

Fig. 1: Computational domain and boundary conditions.

resolution was prescribed in the spanwise direction $(\Delta z)$ in the laminar, transition, and fully developed turbulent flow regimes of the boundary layer. This approach was undertaken to minimize the commutation errors that can otherwise affect the accuracy of an LES computation due to excessive non-homogeneous grid resolution. In the wall-normal direction, $\Delta y$, the boundary layer was resolved with 120 grid cells. Away from the boundary layer region, the wall-normal grid resolution was coarsened by utilizing a separate mesh block. Similarly to the spanwise direction grid design, identical grid resolution was employed in the laminar, transition, and fully developed turbulent flow regions of the boundary layer in the wall-normal direction. The dimensions of the computational grid wall spacing are described in Table 2. The distinction between the grid resolution in the laminar-transition and fully developed turbulent regions is made with subscripts in the streamwise direction grid spacing, where $\Delta x_{B 1}$ and $\Delta x_{B 3}$ are representative of the laminar-transition and fully developed turbulent regions, respectively. $\Delta x_{B 2}$ corresponds to the non-uniform mesh region that merges the laminar-transition and fully developed turbulent flow computational regions. A coarse version of the grids utilized for the numerical computations is depicted in Fig. 2.

In determining the grid size required to resolve the viscous sublayer, $\delta_{V S L}$, a priori estimates of the length scale is not trivial. Such estimates are readily available for momentum-driven turbulent boundary layers at high Reynolds number and buoyancy-driven turbulent boundary layers at high Grashof/Rayleigh number conditions. Assuming a momentum-driven turbulent boundary layer scaling, $\delta_{V S L}$ can be approximated using the wall shear stress, $\tau_{w}$, and the fluid kinematic viscosity, $\nu_{w}[32]$ :

$$
\delta_{V S L} \approx \frac{\nu_{w}}{\left(\tau_{w} / \rho_{w}\right)^{1 / 2}} .
$$

The scaling used to estimate $\delta_{V S L}$ for purely buoyancy-driven turbulent boundary layers employs the nearwall heat flux, $q_{w}$, and the fluid thermal diffusivity, $\nu_{w} / \operatorname{Pr}[15]$ : 


$$
1
$$




\begin{tabular}{|l|c|c|c|c|c|}
\hline Case & $\Delta x_{B 1}$ & $\Delta x_{B 2}$ & $\Delta x_{B 3}$ & $\Delta y$ & $\Delta z$ \\
\hline \hline$R i_{x}=\infty$ & 7 & {$[7,25]$} & 25 & 0.53 & 3.6 \\
\hline$R i_{x}=39.42$ & 3 & {$[3,20]$} & 20 & 0.28 & 2.0 \\
\hline$R i_{x}=15.27$ & 1.5 & 1.5 & 1.5 & 0.19 & 0.6 \\
\hline
\end{tabular}

Table 2: Computational grid wall spacing $(\mathrm{mm})$.

\begin{tabular}{|l|c|c|c|}
\hline Case & $N_{x}$ & $N_{y}$ & $N_{z}$ \\
\hline \hline$R i_{x}=\infty$ & 365 & 149 & 250 \\
\hline$R i_{x}=39.42$ & 908 & 170 & 150 \\
\hline$R i_{x}=15.27$ & 3332 & 191 & 150 \\
\hline
\end{tabular}

Table 3: Largest cell distribution of computational grid.

$$
\delta_{V S L} \approx \frac{\left(\nu_{w} / P r\right)^{3 / 4}}{\left(q_{w} / \rho_{w} c_{p, w}\right)^{1 / 4}(\mathrm{~g} \beta)^{1 / 4}}
$$

The $\delta_{V S L}$ acquired from Eq.(16) was employed to calculate $\Delta y_{w}$ for the $R i_{x}=\infty$ flow configuration. $\Delta x_{w}$ and $\Delta z_{w}$ were determined through an iterative process subsequently explained in brief. Since $\Delta z_{w}$ is invariable in the laminar, transition, and fully turbulent flow regions, $\Delta z_{w}$ was established strictly based on the necessity to resolve the emergence and growth of spanwise vortex structures in the transition region. Thus, the iterative process encompassed initially setting large values for $\Delta z_{w}$ in preliminary computations, and refining its value in succeeding new simulations until the transition region is reasonably captured. A similar approach was utilized to determine the optimum $\Delta x_{w}$ grid spacing in the laminar-transition and fully developed turbulent regions. In the laminar-transition region, the stringent criteria of capturing the initial stage and evolution of streamwise vortex structures were used to ascertain $\Delta x_{w}$. However, in the fully developed turbulent region, $\Delta x_{w}$ can be relaxed since the streamwise vortex structures are larger, thus needing less refinement to effectively resolve the underlying physics. The streamwise direction grid-stretching ratio employed to merge these two regions did not exceed 1.20 in order to capture all the flow physics of interest.

Due to the absence of any available $\delta_{V S L}$ scaling for mixed-convection turbulent boundary layers, the grid resolution for $R i_{x}=39.42$ and $R i_{x}=15.27$ flow configurations in the laminar-transition and fully developed turbulent regions were approximated using the $R i_{x}=\infty$ converged grid spacing as a guideline.

In the process of achieving accurate results efficiently, it was compulsory to run the simulations initially on coarser grids and to map the coarse mesh solution onto successively pre-determined finer grids until the second order turbulent statistics attained statistical convergence. The simulations were conducted by allowing for at least 40 flow-through times on the finest grids, and the solution was time-averaged after the initial transients were flushed through the computational domain which was roughly after 5 flow-through times. A flow-through time was computed by tracking a fluid particle within the boundary layer as it traversed the computational domain from the inflow to the outflow. Subsequent to the completion of the simulations, the time-averaged results were spatially averaged in the homogeneous spanwise direction.

Based on the aforementioned solution procedure, the highest grid density employed for the simulations are summarized in Table $3 . N_{x}, N_{y}$, and $N_{z}$ are indicative of the cell distribution in the streamwise, wall-normal, and spanwise directions of the computational domain.

\section{Results and discussion}

Near-wall profiles of the flow quantities presented in the subsequent subsections will be depicted in linear and semi-logarithmic scales. The linear scale serves the purpose of assessing the far-wall boundary layer region, and the semi-logarithmic scale magnifies the near-wall region of the boundary layer. The near-wall profiles correspond to a streamwise direction location of $x=3.70 \mathrm{~m}$. 


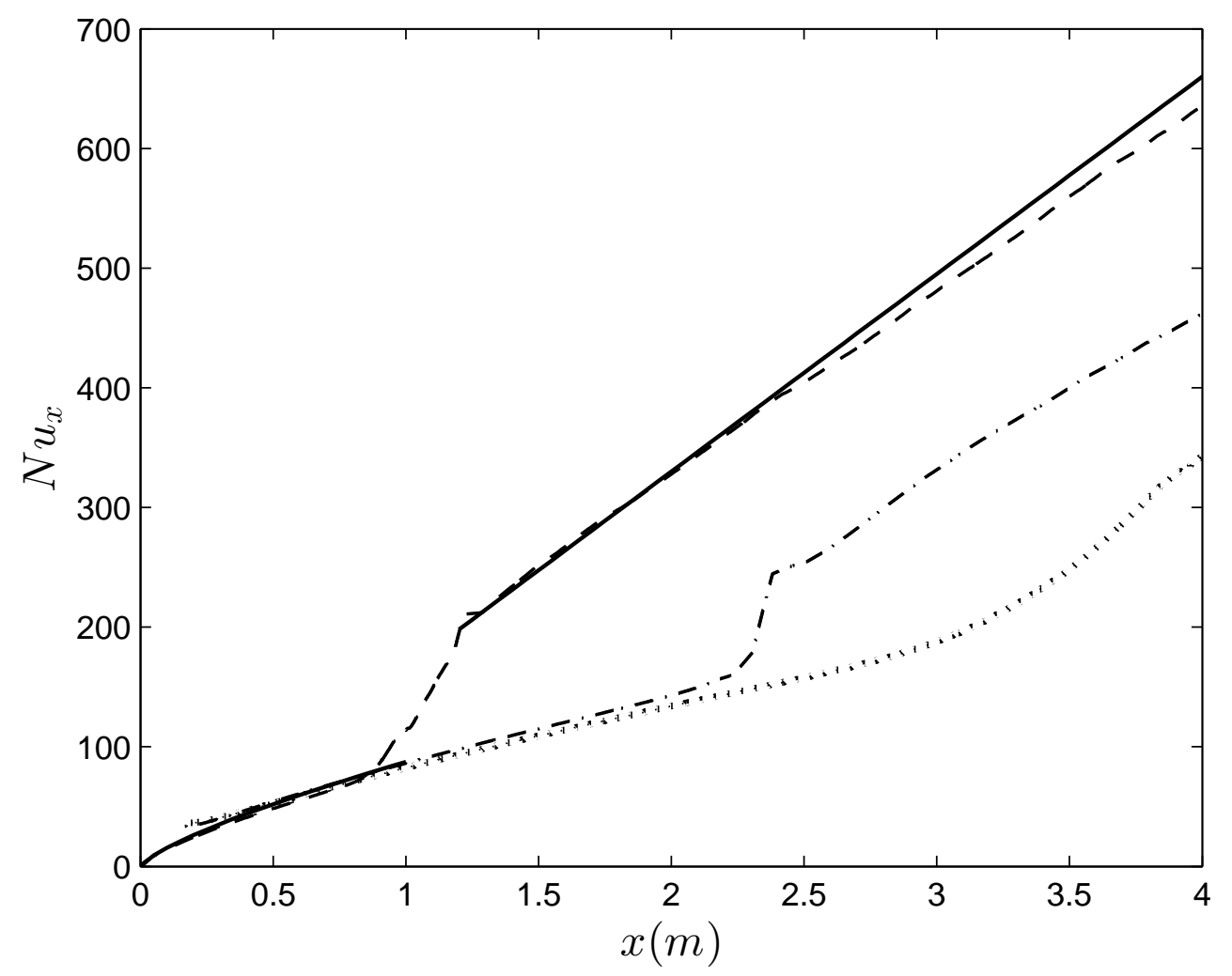

Fig. 3: Wall heat transfer rates. solid line: empirical equation [36], LES results: dashed line: $R i_{x}=\infty$; dashed-dotted line: $R i_{x}=39.42$; dotted line: $R i_{x}=15.27$.

\subsection{Wall heat transfer rates}

Wall heat transfer rates in the streamwise direction are illustrated in Fig. 3. In the absence of experimental data, empirical equations formulated by [36] for laminar and turbulent natural-convection boundary layers and LES results are depicted in Fig. 3. The laminar and turbulent heat transfer empirical equations, respectively reads as

$$
N u_{x}=0.387\left(G r_{x} P r\right)^{1 / 4}
$$

and

$$
N u_{x}=0.120\left(G r_{x} P r\right)^{1 / 3}
$$

For the natural-convection $\left(R i_{x}=\infty\right)$ wall heat transfer rates, there is good agreement between the empirical solution and LES in the laminar and turbulent flow regions. As the freestream flow is increased, $\left(R i_{x}=39.42\right)$, a significant delayed transition to turbulence has emerged in the mixed-convection boundary layer, in addition to a drastic reduction in the turbulent wall heat flux. An observation to note for the $R i_{x}=39.42$ case is the existence of a quasi-constant turbulent wall heat flux similar to the classical turbulent natural-convection boundary layer $\left(R i_{x}=\infty\right)$. By further increasing the freestream flow $\left(R i_{x}=15.27\right)$, the mixed-convection boundary layer starts transitioning to turbulence at the downstream end of the $4 \mathrm{~m}$ plate, along with a further reduction in wall heat flux. From Fig. 3, it can be concluded that the near-wall profiles of $R i_{x}=15.27$ configuration pertains to a transitioning boundary layer, as will be later discussed. 

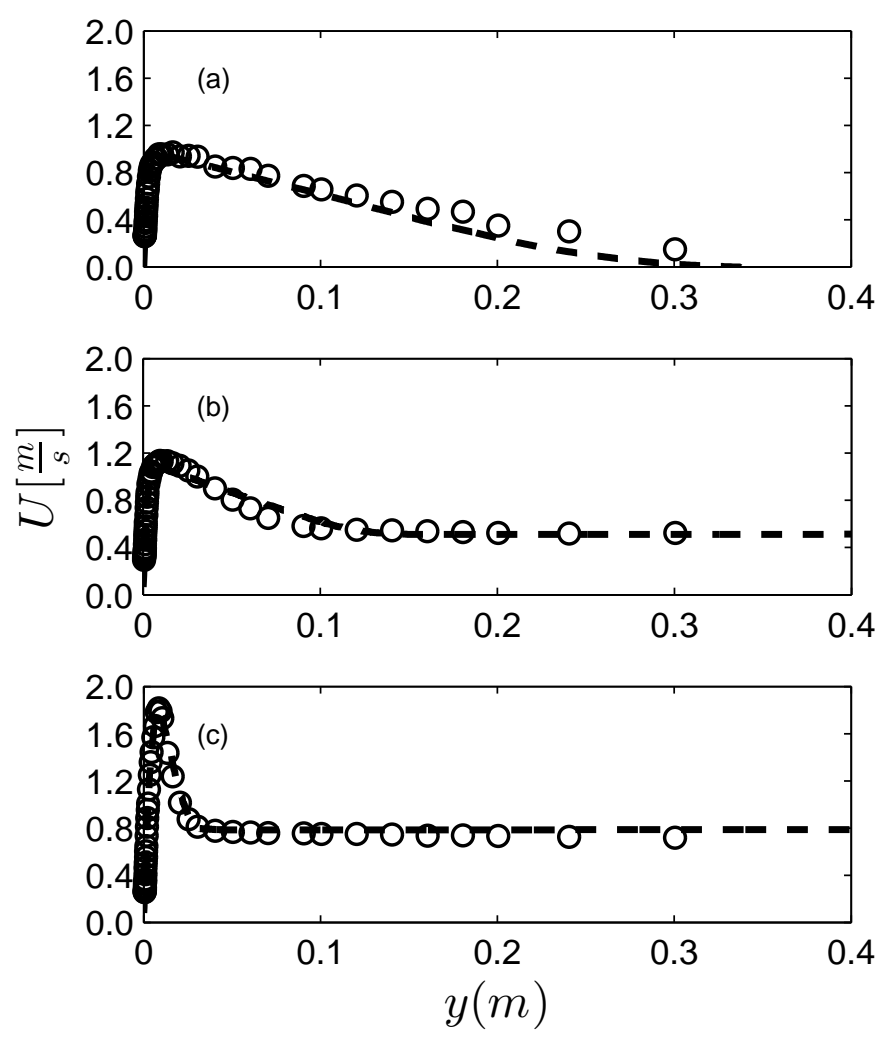

Fig. 4: Mean streamwise velocity profiles in linear scale. Circle: measurements [12], dashed line: LES results. (a) $R i_{x}=\infty$; (b) $R i_{x}=39.42 ;$ (c) $R i_{x}=15.27$.

The capability of the LES to precisely represent the boundary layer transition process "naturally" without the addition of artificial disturbances or turbulence [1-3] should be noted. It can be conjectured that these artificial fluctuations can adversely affect the physics of the transitioning boundary layer and downstream turbulent flow.

\subsection{Mean streamwise velocity and temperature profiles}

The mean streamwise velocity profiles of the experimental measurements and LES are shown in Figs. 4 and 5. There is excellent agreement between the measurements and LES results in the inner and outer layers. It can be seen that the maximum mean streamwise velocity increases with an increased freestream flow, because forced convection effects are augmenting the streamwise velocity in a non-linear manner. Furthermore, the boundary layer progressively becomes thinner as the freestream flow is increased. In Figs. 6 and 7, the mean temperature wall profiles of the measurements and LES are depicted. An interesting thing to note is the increase in wall shear, i.e. $\partial U / \partial y_{w}$, as the freestream flow is increased in the turbulent boundary layer. Usually, turbulence is known to emerge as a result of increasing shear, and the subsequent boundary layer becomes successively more diffusive as the wall shear increases. However, from Fig. 5 , it can be seen that in the presence of increasing wall shear stress, the mixed-convection boundary layer thickness diminishes, and the wall heat transfer decreases as the freestream velocity increases, refer to Table 4 . Thus, it is apparent that the nature of the turbulence generated, either by shear or buoyant production, is being suppressed by some mechanism when the freestream flow is increased, resulting in a less diffusive boundary layer. 

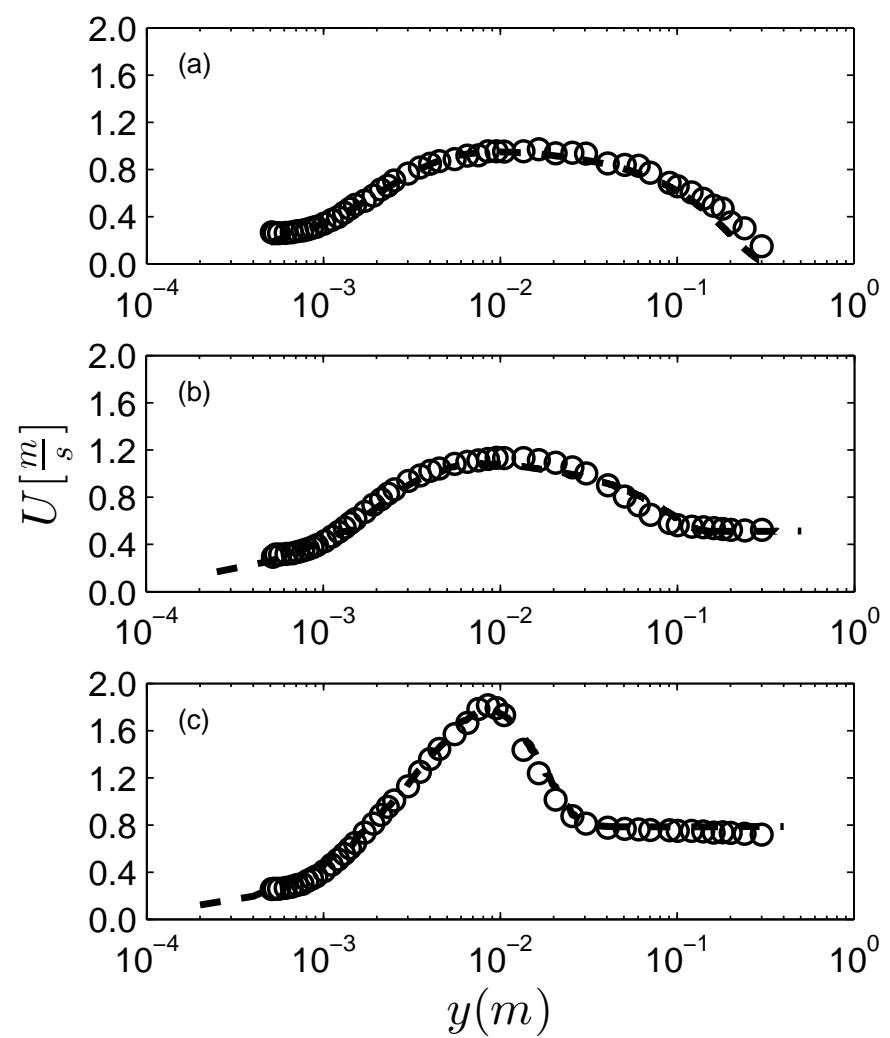

Fig. 5: Mean streamwise velocity profiles in semi-logarithmic scale. Circle: measurements [12], dashed line: LES results. (a) $R i_{x}=\infty ;\left(\right.$ b) $R i_{x}=39.42 ;$ (c) $R i_{x}=15.27$. 

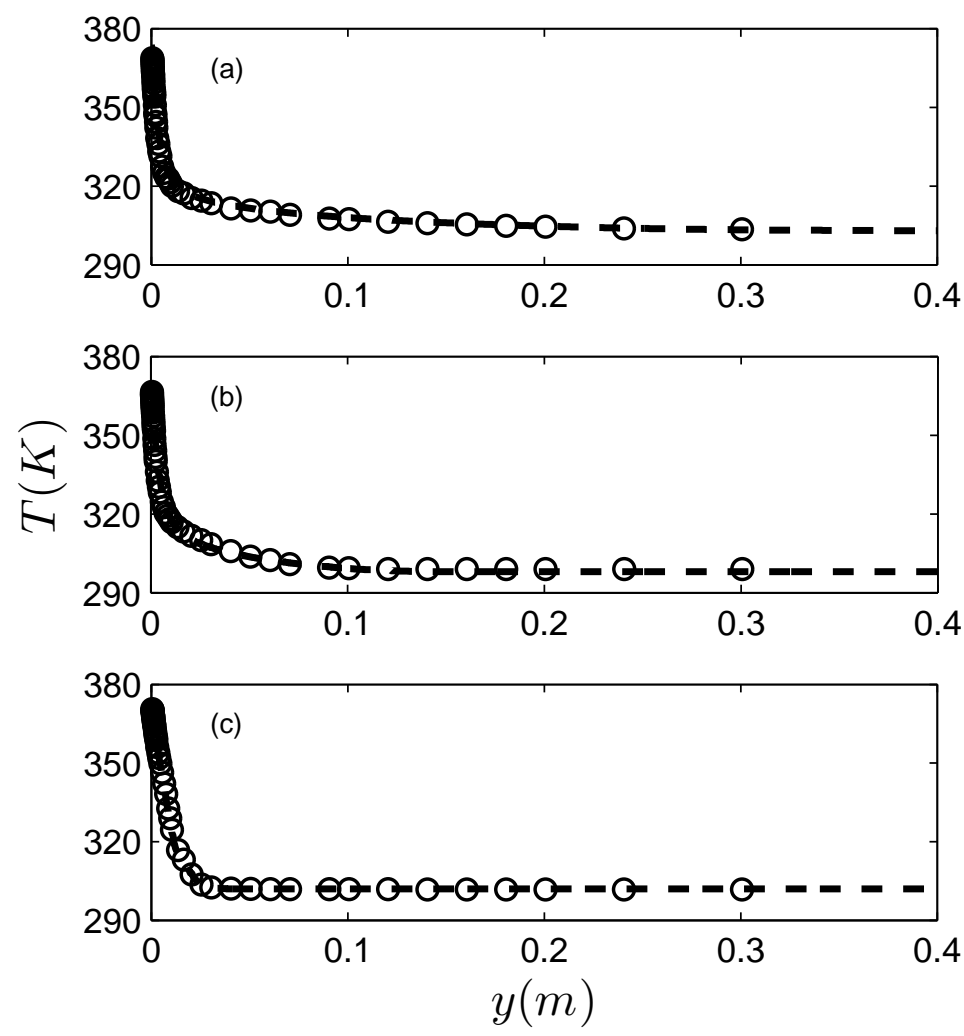

Fig. 6: Mean temperature profiles in linear scale. Circle: measurements [12], dashed line: LES results. (a) $R i_{x}=\infty$; (b) $R i_{x}=39.42 ;(\mathrm{c}) R i_{x}=15.27$. 

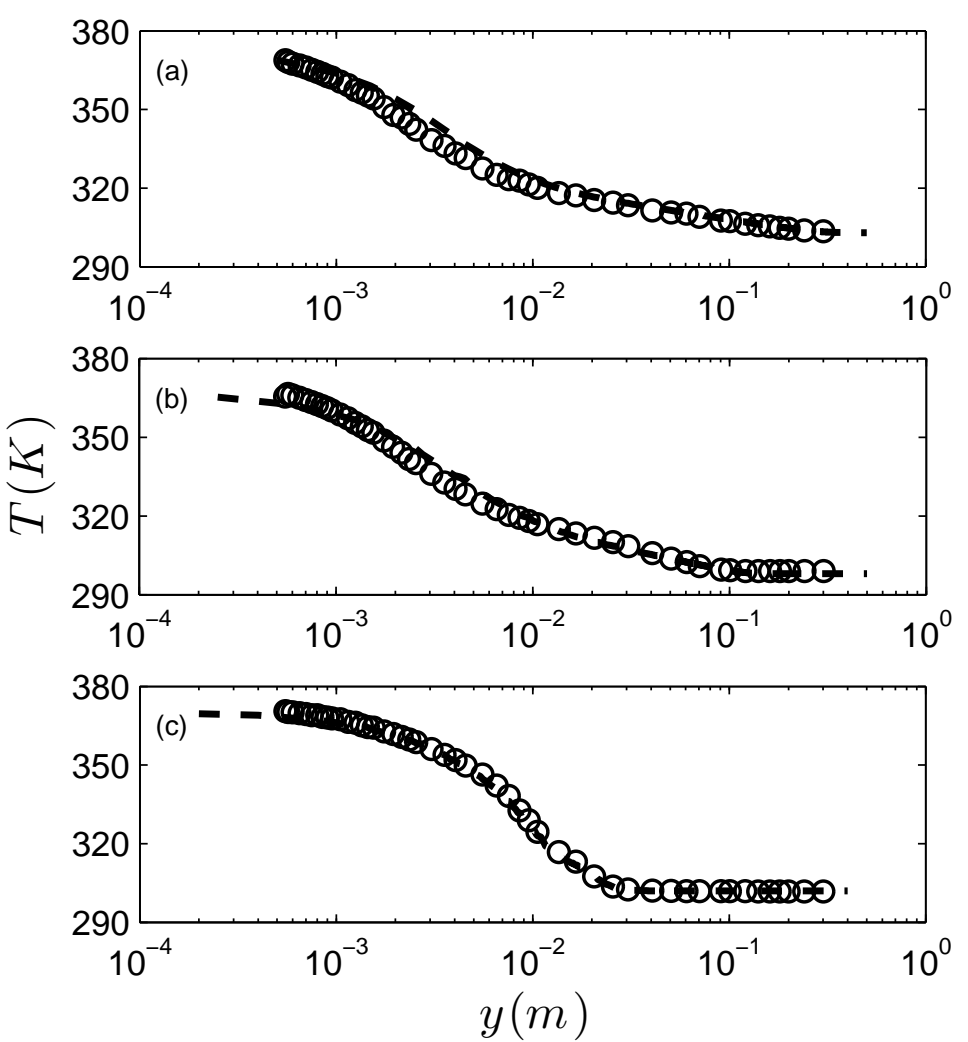

Fig. 7: Mean temperature profiles in semi-logarithmic scale. Circle: measurements [12], dashed line: LES results. (a) $R i_{x}=\infty$; (b) $R i_{x}=39.42$; (c) $R i_{x}=15.27$. 


\begin{tabular}{|l|c|c|}
\hline Case & $q_{w}\left(\frac{W}{\mathrm{~m}^{2}}\right)$ & $\tau_{w}\left(\frac{\mathrm{kg}}{\mathrm{ms}}{ }^{2}\right.$ \\
\hline \hline$R i_{x}=\infty$ & 352.16 & 0.0084 \\
\hline$R i_{x}=39.42$ & 248.08 & 0.0096 \\
\hline$R i_{x}=15.27$ & 168.32 & 0.01 \\
\hline
\end{tabular}

Table 4: Heat transfer and wall shear stress values at streamwise location of $x=3.70 \mathrm{~m}$.

To demonstrate the LES computation for the $R i_{x}=\infty$ configuration is wall-resolved, Eqs. 16 and 18 were utilized to calculate the thickness of the viscous sublayer, $\delta_{V S L}$. The $\delta_{V S L}$ value acquired using Eqs. 16 and 18 was $1.29 \mathrm{~mm}$. Using the natural-convection scaling provided by [15], we define a non-dimensional wall unit $y^{*}=\Delta y_{\text {wall }} / \delta_{V S L} \leqslant 1$, to illustrate the simulation is wall-resolved. The non-dimensional wall unit, $y^{*}=0.53 / 1.29=0.410$, indicates this particular computation is wall-resolved.

For the mixed-convection flow configurations, $R i_{x}=39.42$ and $R i_{x}=15.27$, non-dimensional wall units cannot be computed since such parameters have not been established in literature for these flows. Furthermore, using either the forced- or natural-convection non-dimensional wall unit equations would be inappropriate since pertinent physics relevant to the flow-field would be neglected and because the turbulent characteristics of mixed-convection boundary layer are distinct from both forced- and natural-convection boundary layers [14].

\subsection{Intensities of streamwise velocity and temperature fluctuations and turbulent heat flux}

The intensity of streamwise velocity fluctuations profiles are depicted in Figs. 8 and 9, and the agreement between the experimental data and LES results are remarkable in the inner- and outer layers of the boundary layer. By increasing the freestream flow, $R i_{x}=39.42$, the magnitude of the streamwise velocity fluctuation intensity attains a value less than the $R i_{x}=\infty$ case. Furthermore, the intensity of the velocity fluctuation has diminished in the entire boundary layer region, and it is confined to a thinner wall-normal region. Figs. 10 and 11 illustrate the temperature fluctuation intensities, and freestream effects can be seen by the reduction of temperature fluctuations in the boundary layer region. In Figs. 9c and 11c, increasingly diminishing velocity and temperature fluctuation intensities can be seen by further increasing the freestream. A peculiar doublepeak can be seen in the velocity fluctuation intensity profile measurement, see Fig. 9c. The LES result was able to capture this phenomenon, although the initial peak was marginally underpredicted. The appearance of the double peak in the velocity fluctuation intensity profile was described to be indicative of a beginning transition to turbulence of a mixed-convection boundary layer [5, 22]. Thus, at $R i_{x}=15.27$, the mixedconvection boundary layer did not attain a fully developed turbulent state, and it is a direct consequence of the increased forced flow. It should be noted that the difference in the peak of turbulence intensity fluctuation is significantly pronounced for temperature fluctuation as the freestream flow is increased, see Figs. 9 and 11. The magnitude of the temperature intensity fluctuation at $R i_{x}=15.27$ is nearly $50 \%$ of the $R i_{x}=\infty$ turbulent boundary layer. Hence, it may be conjectured that in this range of mixed-convection boundary layer flow regime, buoyant production of turbulence might be dominant over shear production. It is apparent that increasing the forced flow suppresses these temperature fluctuations, thereby reducing the overall turbulence of the flow. Another interesting observation from Fig. 11c is the appearance of a double peak in the experimental temperature fluctuation intensity. The LES prediction was unable to capture this transitioning phenomenon; similarly, the DNS result of [2] was unable to replicate this behavior in their simulations.

Wall profiles of the streamwise turbulent heat flux, $\overline{u^{\prime} t^{\prime}}$, are depicted in Figs. 12 and 13. The streamwise turbulent heat flux is a measure of the correlation between $u^{\prime}$ and $t^{\prime}$. At $R i_{x}=\infty$ and $R i_{x}=39.42$, there is good agreement between the experimental data and LES predictions in the inner and outer layers of the boundary layer. At the edge of the inner and outer layers, the turbulent heat flux was minimally overestimated by the LES. At $R i_{x}=15.27$, the LES was able to resolve the streamwise turbulent heat flux in the outer layer. However, the LES was unable to accurately resolve the streamwise turbulent heat flux near the wall. This discrepancy was attributed to the scarcity of grid cells within the transitioning boundary layer 

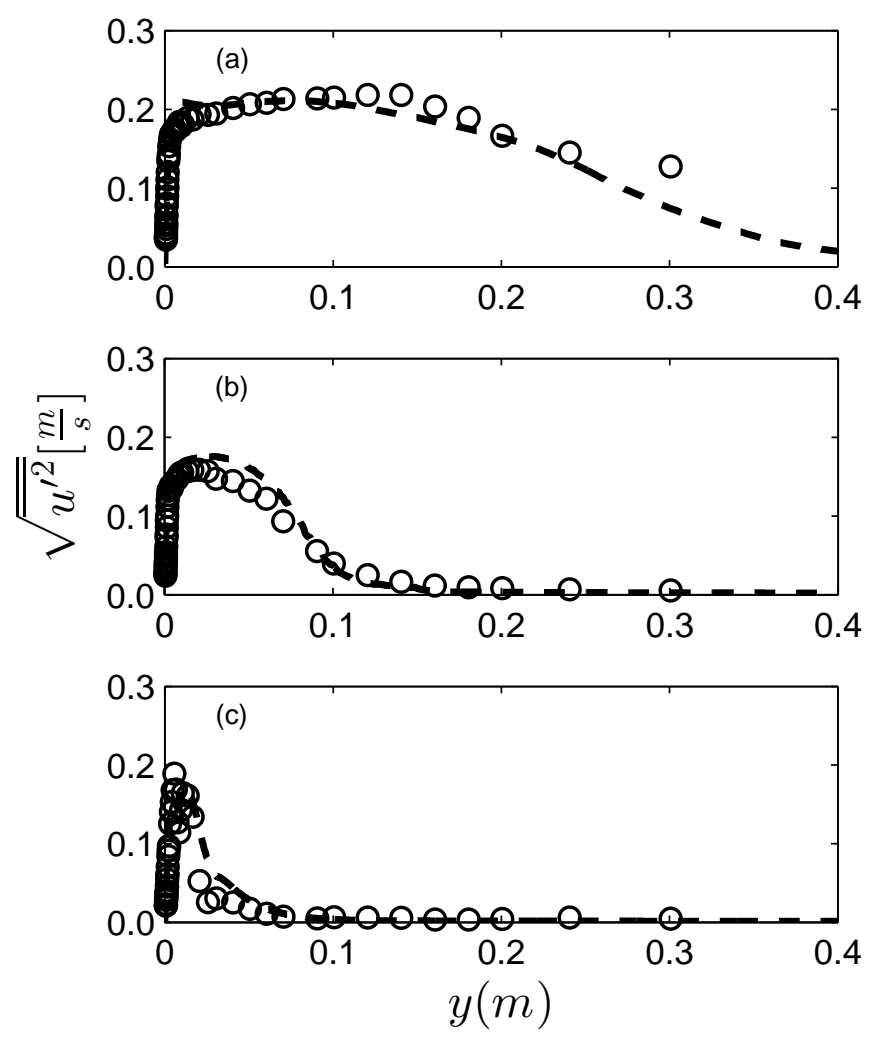

Fig. 8: Streamwise velocity fluctuation intensity profiles in linear scale. Circle: measurements [12], dashed line: LES results. (a) $R i_{x}=\infty$; (b) $R i_{x}=39.42$; (c) $R i_{x}=15.27$. 

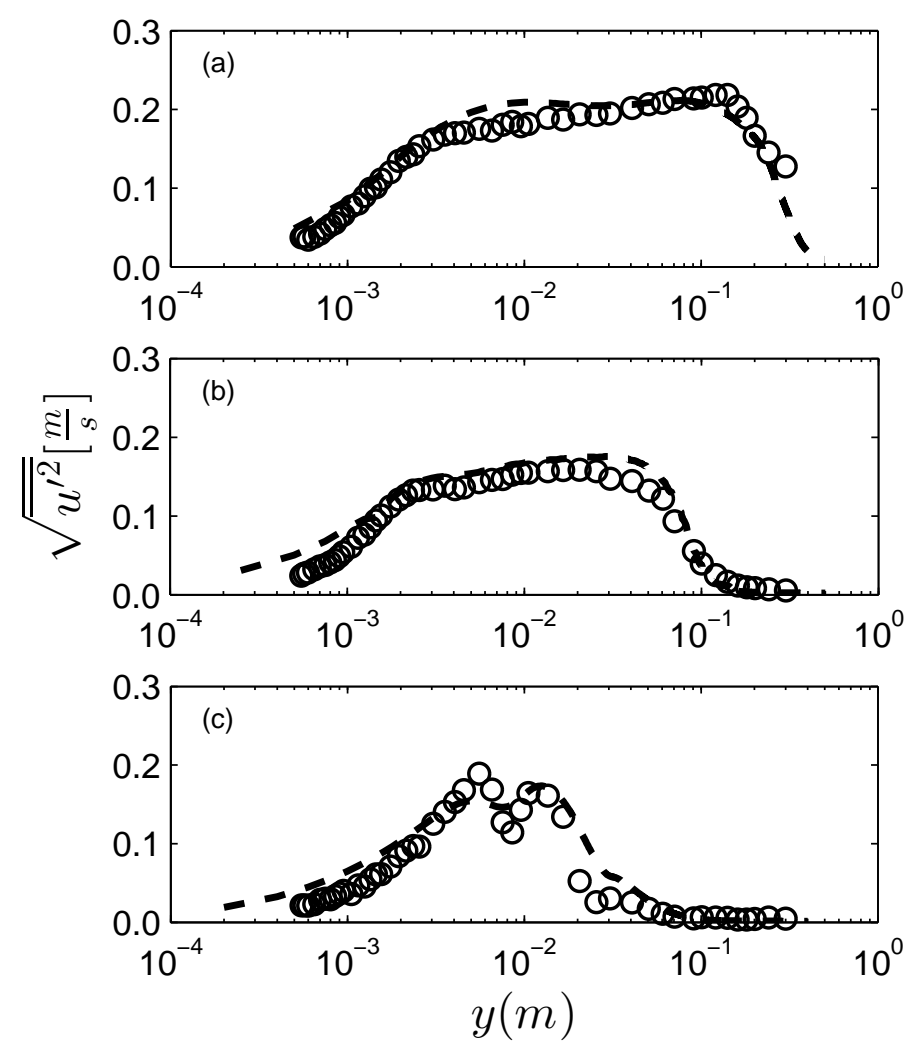

Fig. 9: Streamwise velocity fluctuation intensity profiles in semi-logarithmic scale. Circle: measurements [12], dashed line: LES results. (a) $R i_{x}=\infty$; (b) $R i_{x}=39.42$; (c) $R i_{x}=15.27$. 

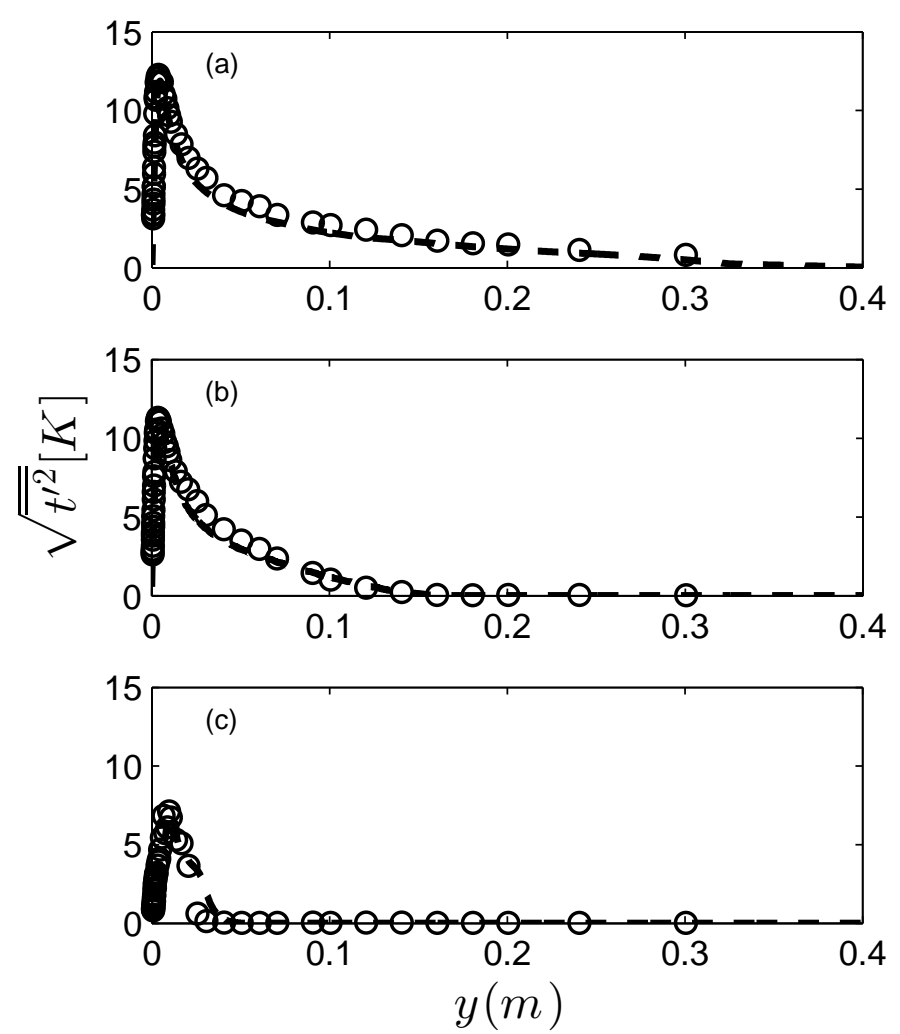

Fig. 10: Temperature fluctuation intensity profiles in linear scale. Circle: measurements [12], dashed line: LES results. (a) $R i_{x}=\infty ;(\mathrm{b}) R i_{x}=39.42$; (c) $R i_{x}=15.27$. 

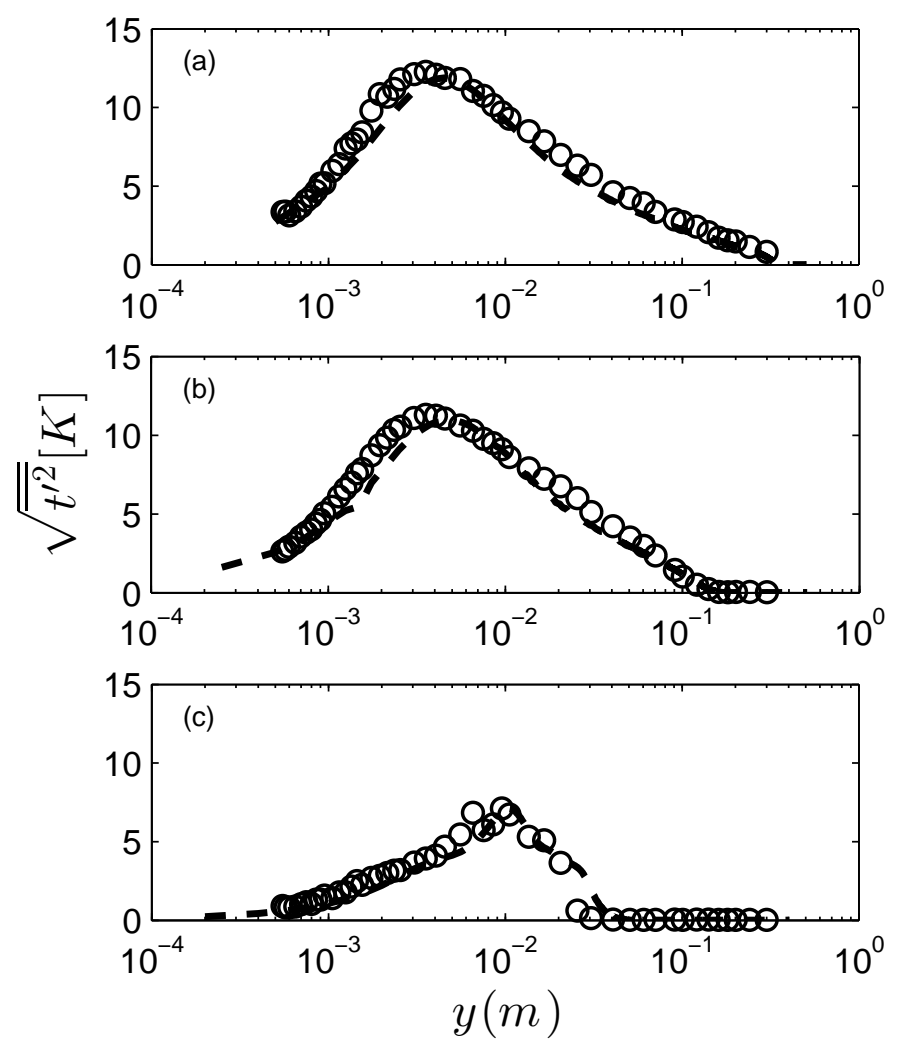

Fig. 11: Temperature fluctuation intensity profiles in semi-logarithmic scale. Circle: measurements [12], dashed line: LES results. (a) $R i_{x}=\infty$; (b) $R i_{x}=39.42$; (c) $R i_{x}=15.27$. 

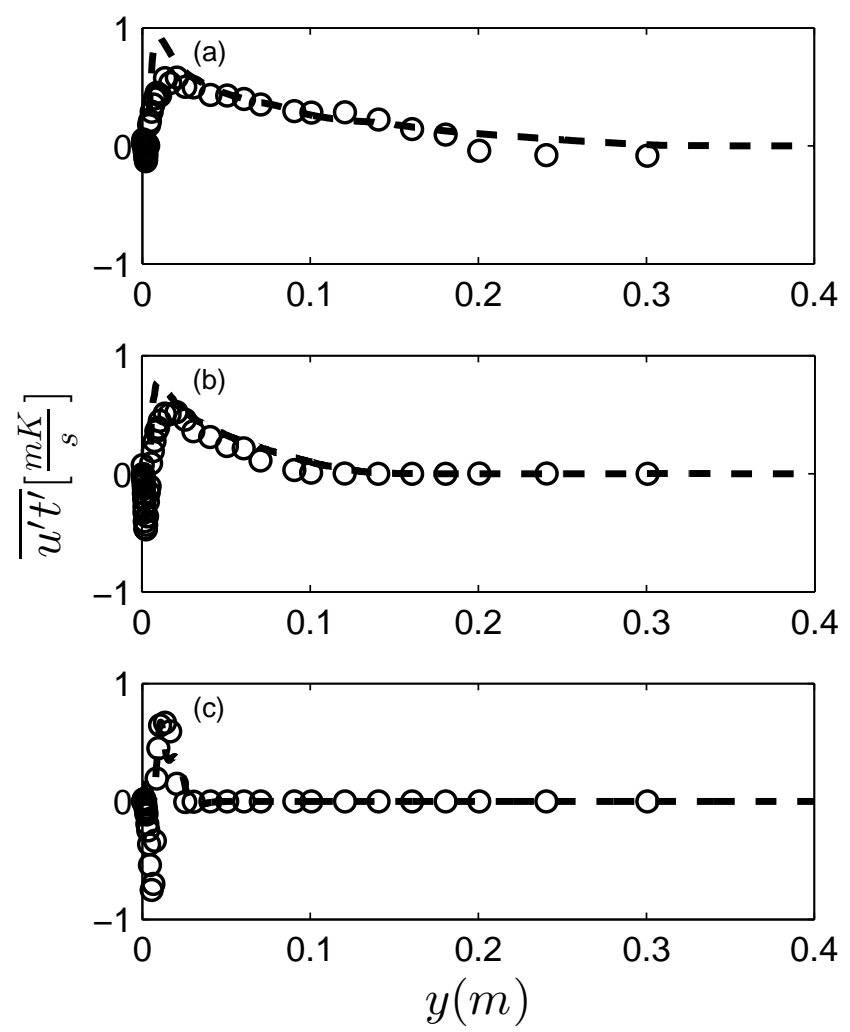

Fig. 12: Streamwise turbulent heat flux profiles in linear scale. Circle: measurements [12], dashed line: LES results. (a) $R i_{x}=\infty ;(\mathrm{b}) R i_{x}=39.42 ;$ (c) $R i_{x}=15.27$.

needed to resolve the intricate transition flow dynamics. Therefore, resolving the transitioning boundary layer was deemed too prohibitively expensive.

An interesting phenomenon to note is the increasingly negative value of $\overline{u^{\prime} t^{\prime}}$ in the inner layer. It can be seen from the measurements this trend becomes more pronounced with an increase in freestream flow, and the LES accurately captured this phenomenon for $R i_{x}=\infty$ and $R i_{x}=39.42$. This occurrence in the inner layer can be due to an influx of either low speed fluid, $u^{\prime}<0$ or low temperature fluid, $t^{\prime}<0$. Analyses of the probability density functions (PDF) of $t^{\prime}$ and $u^{\prime}$ in the inner layer illustrated the PDF of $t^{\prime}$ is largely skewed towards negative, indicating an influx of low temperature fluid in the inner layer region of the turbulent boundary layer. Furthermore, from the inversely proportional relationship between density and temperature, it can be concluded that the negative values of $\overline{u^{\prime} t^{\prime}}$ in the inner layer is similarly due to advection of low density fluctuations, $\rho^{\prime}<0$, in the near-wall region.

The authors wish to emphasize the significance of the achieved agreement between the experiment and LES for the streamwise turbulent heat flux, $\overline{u^{\prime} t^{\prime}}$, in the near-wall region. None of the prior DNS, LES, or RANS studies $[1-3,35]$ have been able to illustrate such detailed agreement as a result of the complexities inherent to mixed-convection turbulent boundary layer flows.

\subsection{Waveforms and Spectra of streamwise velocity and temperature fluctuations}

Waveforms of temperature and velocity fluctuations are illustrated in Figs. 14 and 15. To capture the temperature and velocity time-series data, computational probes were implemented at a streamwise direction location of $x=3.70 \mathrm{~m}$. The probes were placed at the center of the simulation domain in the spanwise 

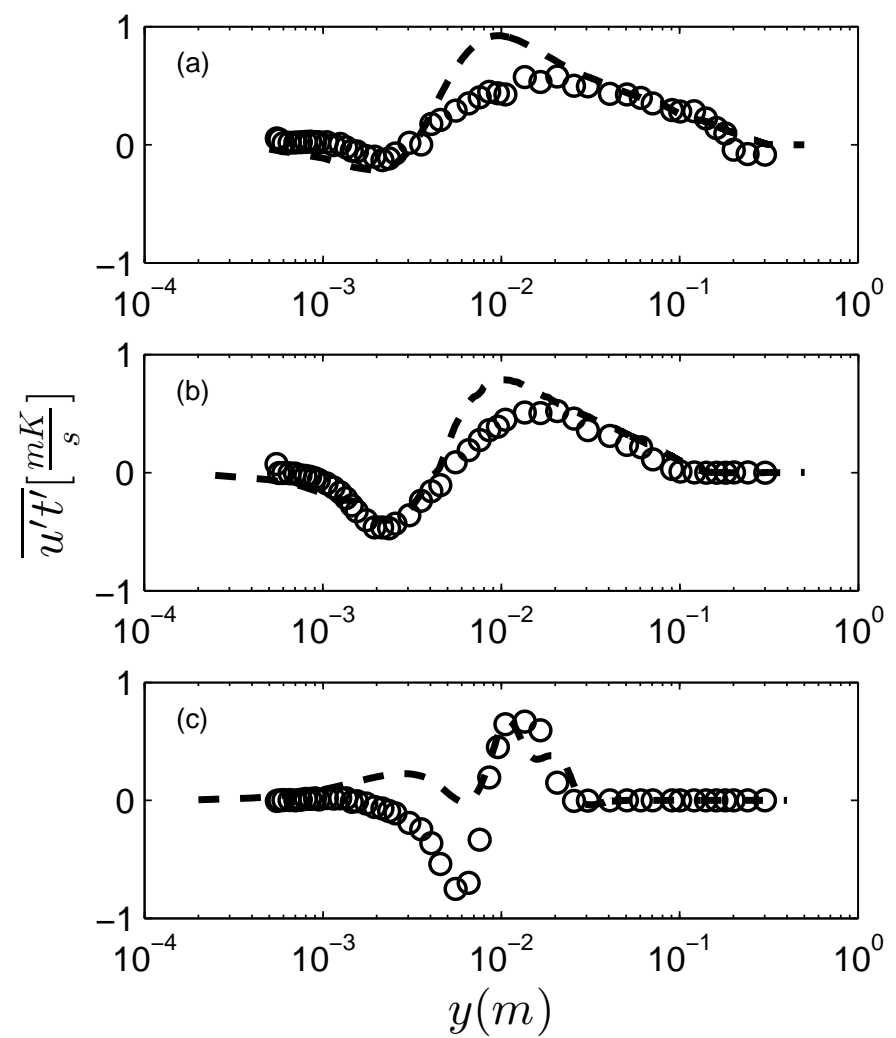

Fig. 13: Streamwise turbulent heat flux profiles in semi-logarithmic scale. Circle: measurements [12], dashed line: LES results. (a) $R i_{x}=\infty$; (b) $R i_{x}=39.42$; (c) $R i_{x}=15.27$. 

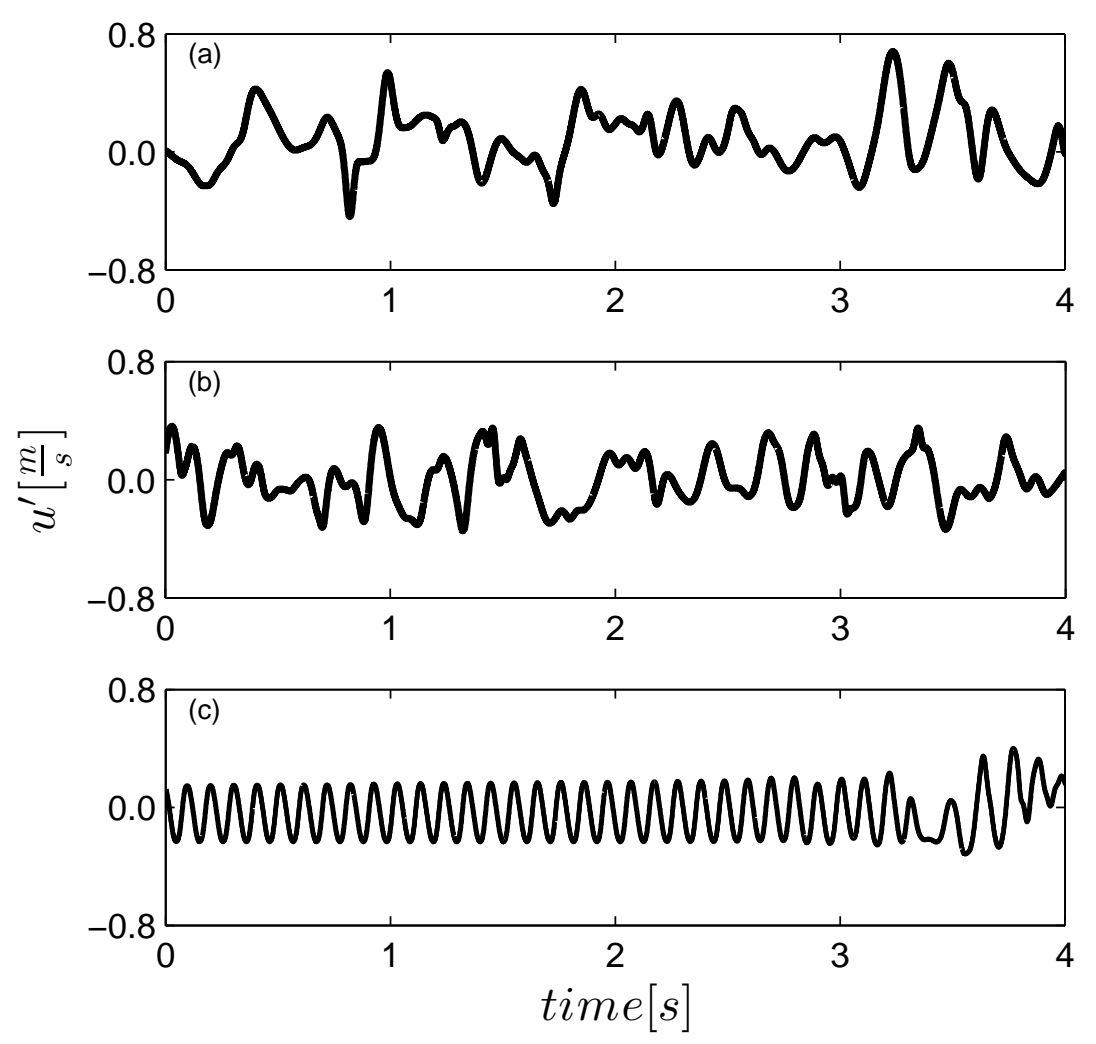

Fig. 14: Waveforms of streamwise velocity fluctuation in the outer layer acquired from LES. (a) $R i_{x}=\infty$; (b) $R i_{x}=39.42$; (c) $R i_{x}=15.27$.

direction and a wall-normal location corresponding to the outer layer where the mean velocity acquires an intermediate value between the maximum and freestream velocity values.

The waveforms given in Figs. 14 and 15 illustrate that large scale fluid motion, indicative of the long period waves, dominate in the outer layer for $R i_{x}=\infty$. However, by increasing the freestream flow, $R i_{x}=39.42$, large scale eddies of higher frequencies now dominate the outer layer. By increasing the freestream flow to $R i_{x}=15.27$, harmonic fluid motion at a single frequency is dominant in the outer layer. The harmonic fluid motion seen in Figs. 14c and 15c might be two-dimensional Tollmien-Schlichting transition waves traveling in the streamwise direction, and the aperiodicity observed at the end of the signal can be attributed to turbulent spots passing by to create temporary turbulent signals.

To further assess the waveforms shown in Figs. 14 and 15, the frequency spectra of streamwise velocity and temperature fluctuations in the outer layer for the measurements and LES computations are illustrated in Figs. 16 and 17, respectively. For the $R i_{x}=\infty$ case, the measured data and LES show large scale eddies of approximately $1 \mathrm{~Hz}$ are the most energetic fluid motion present in the outer layer. It is also apparent that eddies of frequencies lower and higher than $1 \mathrm{~Hz}$ are also in motion in this region. At $R i_{x}=39.42$, eddies of varying frequencies exist in the outer layer region; however, the dominant frequency is around 3 Hz. Thus, it is apparent that with an increase in freestream flow, the larger scale eddies in the outer layer responsible for most of the momentum and heat transfer are being suppressed. This is further evident by examining the frequency spectra in the outer layer at $R i_{x}=15.27$. From Figs. $15 \mathrm{c}$ and $16 \mathrm{c}$, it can be seen that random fluid motion was markedly nonexistent, and harmonic fluid motion is dominant at a specific fundamental frequency. We can see that this fundamental frequency is around $11 \mathrm{~Hz}$. 

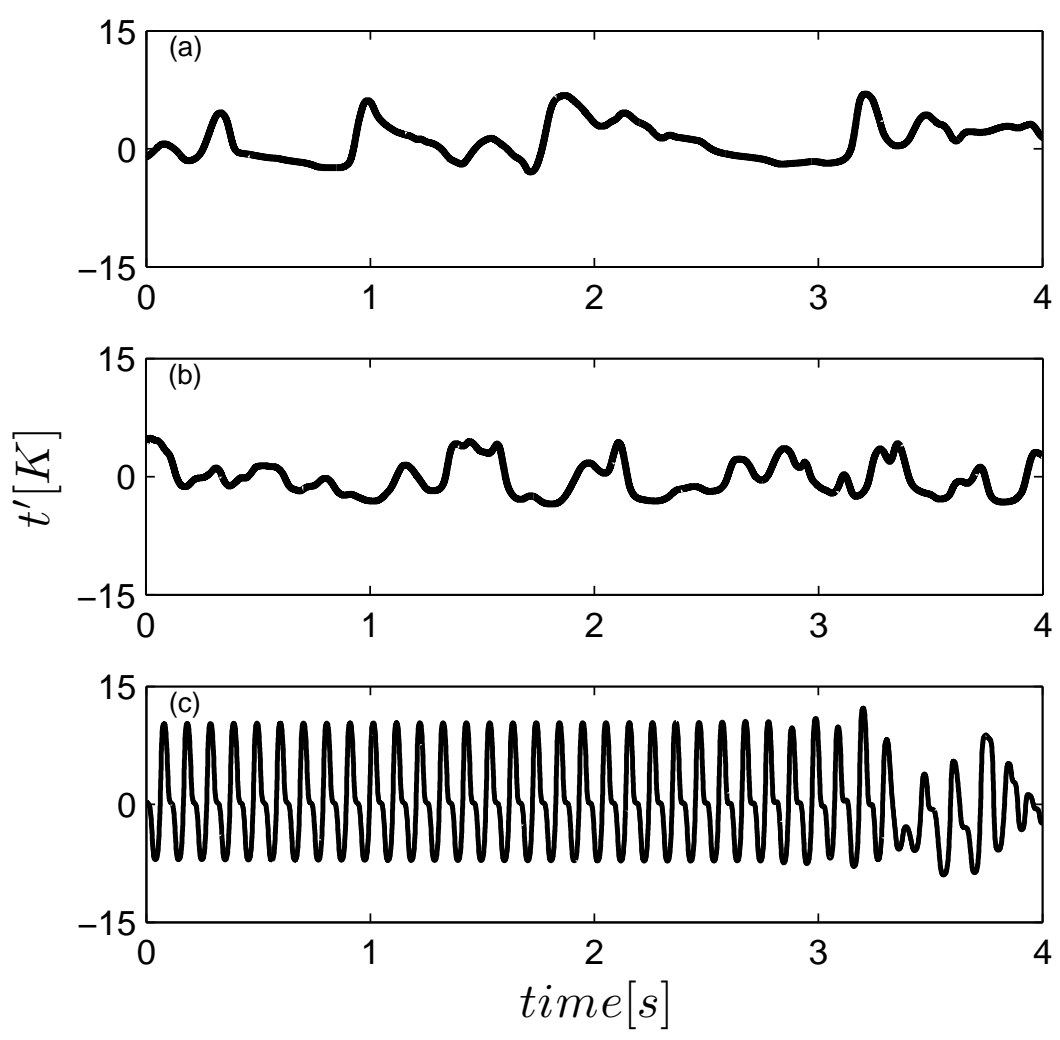

Fig. 15: Waveforms of temperature fluctuation in the outer layer acquired from LES. (a) $R i_{x}=\infty$; (b) $R i_{x}=39.42$; (c) $R i_{x}=15.27$. 

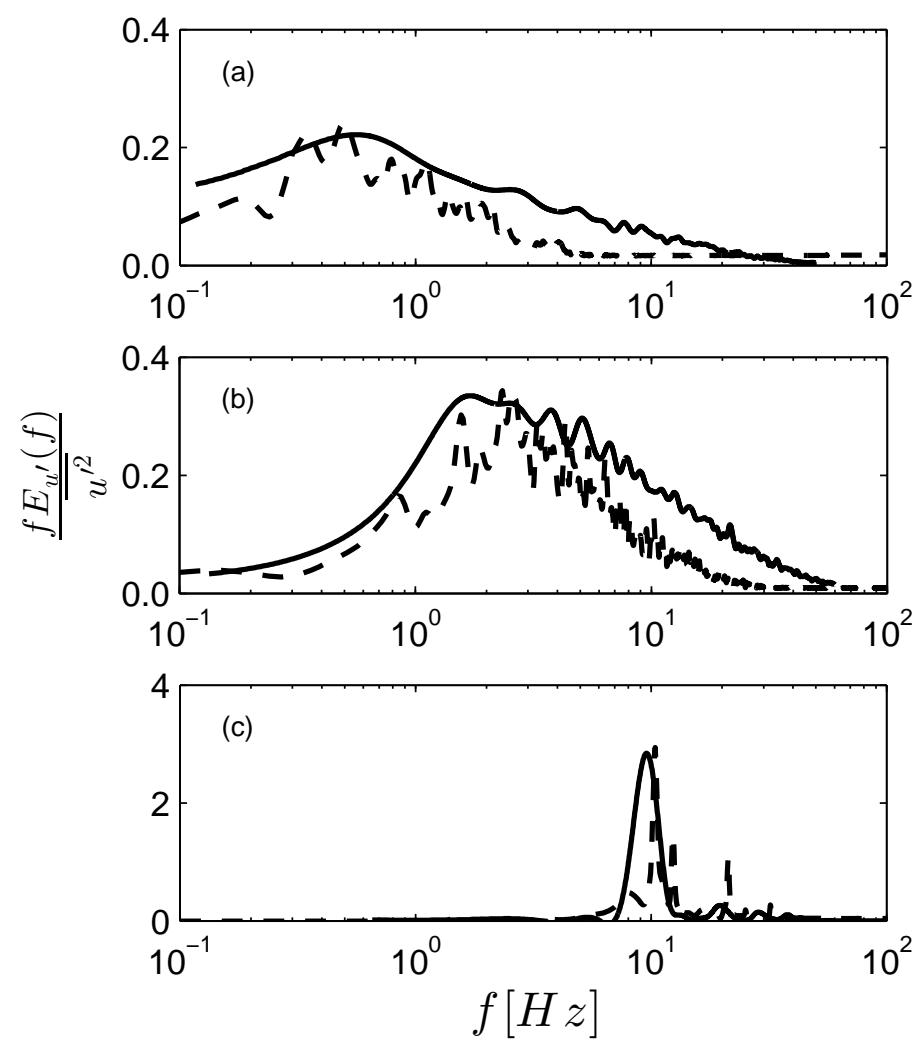

Fig. 16: Frequency spectra of streamwise velocity fluctuation in the outer layer. Dashed line: measurements [12], solid line: LES results. (a) $R i_{x}=\infty$; (b) $R i_{x}=39.42$; (c) $R i_{x}=15.27$. 

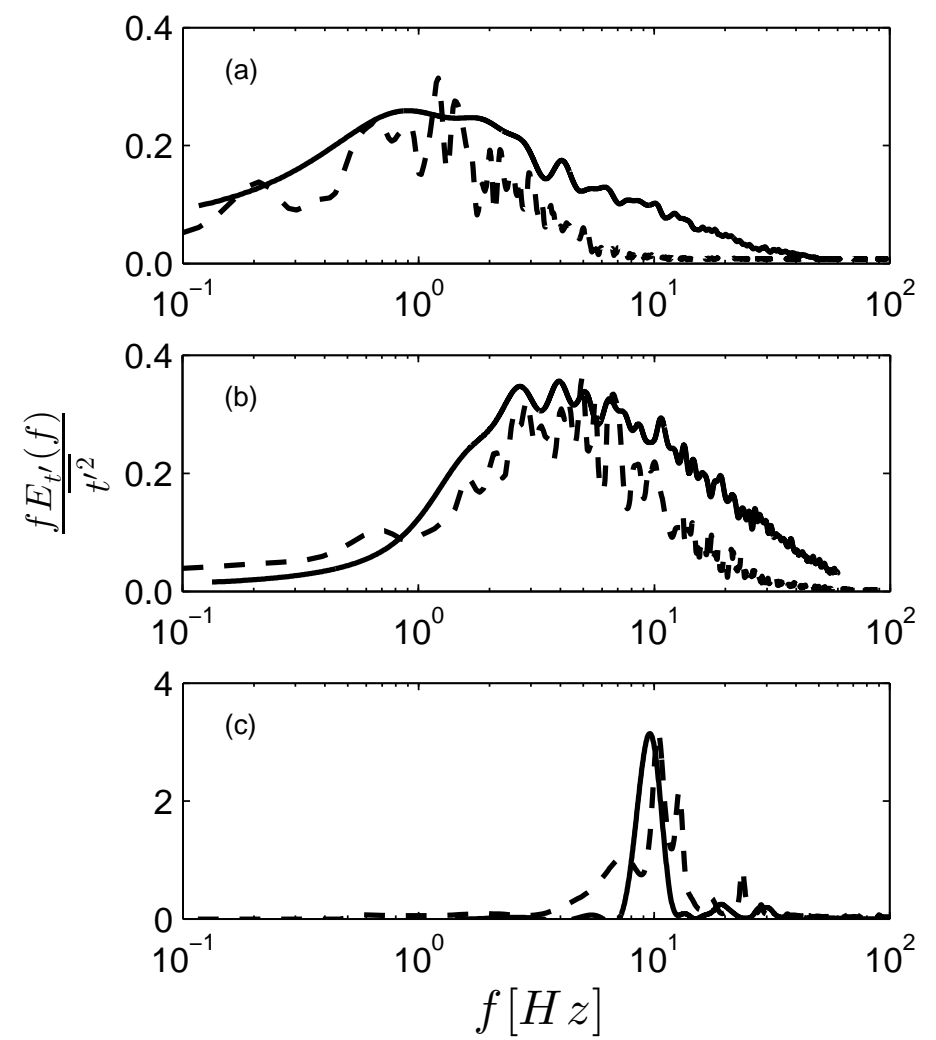

Fig. 17: Frequency spectra of temperature fluctuation in the outer layer. Dashed line: measurements [12], solid line: LES results. (a) $R i_{x}=\infty$; (b) $R i_{x}=39.42$; (c) $R i_{x}=15.27$. 

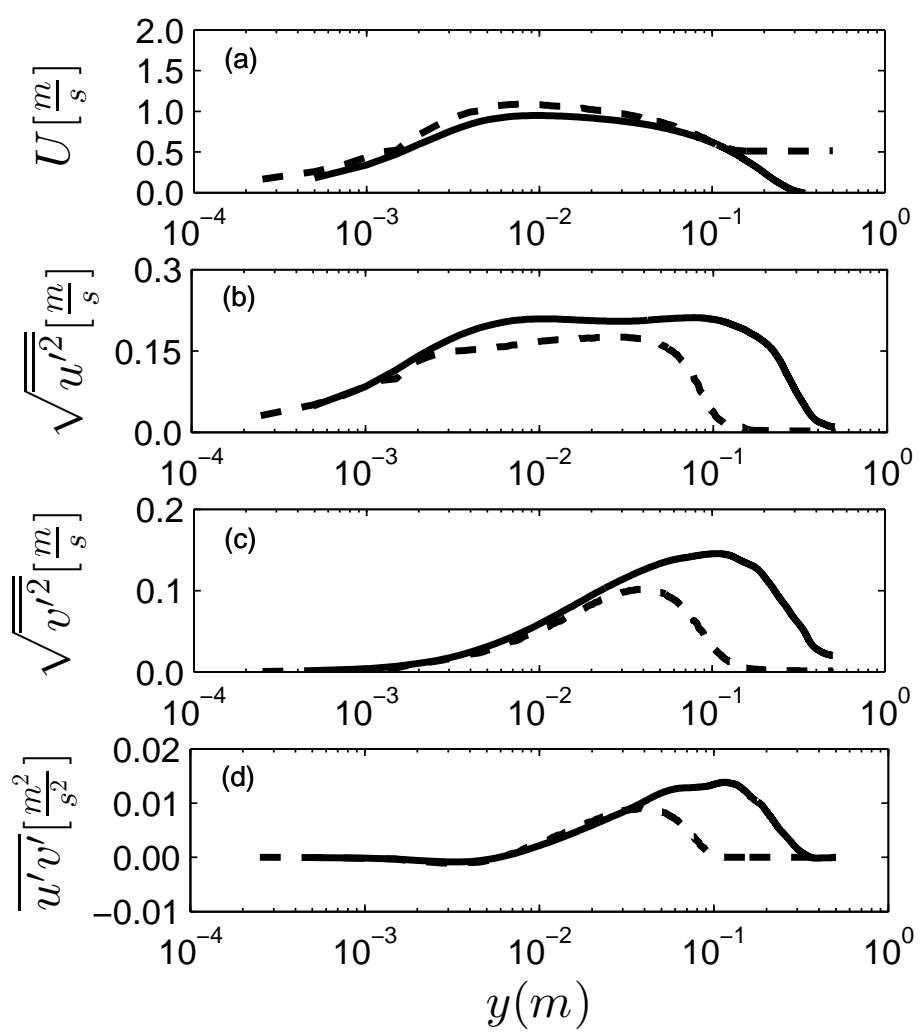

Fig. 18: Structural characteristics of mixed-convection velocity field. Solid line: $R i_{x}=\infty$, dashed line: $R i_{x}=39.42 ;(a)$ Mean streamwise velocity; (b) Streamwise velocity fluctuation intensity; (c) Wall-normal velocity velocity fluctuation; (d) Reynolds shear stress.

\subsection{Structural characteristics of the velocity field}

The inner and outer layer characteristics of the mixed-convection turbulent boundary layer will be subsequently assessed. Fig. 18a depicts the mean streamwise velocity at $R i_{x}=\infty$ and $R i_{x}=39.42$. The streamwise and wall-normal velocity fluctuation intensities at $R i_{x}=\infty$ and $R i_{x}=39.42$ can be seen in Figs. $18 \mathrm{~b}$ and 18c, respectively. In Fig. 18d, the Reynolds shear stress profile can be seen to attain its maximum value in the outer layer. It is interesting to note that in the inner layer, near the wall, $\overline{u^{\prime} v^{\prime}}$ is essentially 0 , although it attains a slightly negative value near the edge of the inner layer. Furthermore, from Fig. 18a, the mean streamwise velocity gradient near the wall is greater than 0 . Thus, in the presence of shear, the Reynolds shear stress near the wall is zero in a mixed-convection turbulent boundary layer. The production of turbulent kinetic energy near the wall is dominated by sweeps, $u^{\prime}>0, v^{\prime}<0$, in wall bounded forced convection turbulent flows [4]. These sweeps are a profound contributor to Reynolds shear stress producing events, and it can be readily concluded from the above discussion that such localized turbulence producing events are not found in the near wall region of a mixed-convection turbulent boundary layer. Hence, it can be concluded that the generation of turbulence in the inner layer may be driven primarily by buoyant turbulent production, $g \beta \overline{u^{\prime} t^{\prime}}$.

At the wall-normal location where the mean streamwise velocity reaches its maximum value, i.e. $\partial U / \partial y=$ $0, \overline{u^{\prime} v^{\prime}}>0$. The occurrence of this phenomenon in the absence of significant shear can be caused by the dominance of positive amplitude fluctuations of $u^{\prime} v^{\prime}$. Thus, the production of Reynolds shear stress in this region can be ascribed to the temperature fluctuations (buoyant production) in the boundary layer. In the 
outer layer of the boundary layer, we see that the Reynolds shear stress continues to increase and acquires its highest value in that region, see Fig. 18d. In addition, from Fig. 18a, $\partial U / \partial y$ becomes increasingly less than zero. As such, the conclusion can be drawn that the presence of substantial shear in addition to buoyant turbulent production in the outer layer contributes to the production of Reynolds shear stress. Therefore, in the outer layer of the mixed-convection turbulent boundary layer, a behavior similar to that of the forced convection turbulent boundary layer exists, i.e., $\overline{u^{\prime} v^{\prime}}>0$ when $\partial U / \partial y<0$.

\section{Conclusions}

The fluid flow and heat transfer characteristics of buoyancy affected turbulence in mixed-convection boundary layers were assessed with the utilization of wall-resolved large eddy simulation. The numerical simulations were performed using lowMachFOAM [28], and the results were compared with the experimental data previously obtained by [12]. The wall-resolved LES of mixed-convection turbulent boundary layer flow over an isothermal vertical flat plate has led to some pertinent conclusions that will be subsequently summarized:

(1) In addition to the excellent agreement observed between the LES and experimental measurements in the inner and outer layers, the LES precisely resolved the rapid changes in the turbulent boundary layer as the freestream velocity is increased by capturing the preceding transition region without artificial or synthetic turbulence.

(2) Until the present study, none of the prior DNS, LES, or RANS computations have been able to show such detailed near-wall agreement between the experimental and numerical data for the streamwise turbulent heatflux, $\overline{u^{\prime} t^{\prime}}$.

On the basis of the present work, the authors plan to provide further studies of the near-wall flow dynamics by investigating the characteristics of the spatio and temporal structures of near-wall coherent structures, appropriate scaling for the turbulence statistics, and other turbulence parameters pertinent to the region of interest.

\section{Acknowledgements}

The numerical simulations presented were made available within the framework of the United States Department of Defense High Performance Computing Modernization Program (HPCMP), for which the authors express immense gratitude.

\section{References}

[1] Abedin, M., Tsuji, T., Hattori, Y., 2009. Direct numerical simulation for a time-developing natural-convection boundary layer along a vertical flat plate. International Journal of Heat and Mass Transfer 52, 4525-4534.

[2] Abedin, M., Tsuji, T., Hattori, Y., 2010. Direct numerical simulation for a time-developing combined-convection boundary layer along a vertical flat plate. International Journal of Heat and Mass Transfer 53, 2113-2122.

[3] Barhaghi, D., Davidson, L., Karlsson, R., 2006. Large-eddy simulation of natural convection boundary layer on a vertical cylinder. International Journal of Heat and Fluid Flow 27, 811-820.

[4] Bernard, P., Wallace, J., 2002. Turbulent Flow: Analysis, Measurement, and Prediction. John Wiley and Sons, New York, USA.

[5] Carey, V., Gebhart, B., 1983. The stability and disturbance amplification characteristics of vertical mixed convection flows. Journal of Fluid Mechanics 127, 185-201.

[6] Choi, Y.-H., Merkle, C., 1993. The application of preconditioning in viscous flows. Journal of Computational Physics 105, 207-223.

[7] Erlebacher, G., Hussani, M., Speziale, C., Zang, T., 1992. Toward the large-eddy simulation of compressible turbulent flows. Journal of Fluid Mechanics 238, 155-185.

[8] Fureby, C., Tabor, C., Weller, H., Gosman, A., 1997. A comparative study of subgrid scale models in homogenous isotropic turbulence. Physics of Fluids 9, 1416-1429.

[9] Garnier, E., Adams, N., Sagaut, P., 2009. Large Eddy Simulation for Compressible Flows. Springer, New York, USA.

[10] Germano, M., 1992. Turbulence: the filtering approach. Journal of Fluid Mechanics 238, 325-336. 
[11] Hall, W., Price, P., 1970. Mixed forced and free convection from a vertical heated plate to air. In: Mixed forced and free convection from a vertical heated plate to air. Vol. 4 of 3.3. Proceedings of the Fourth International Heat Transfer Conference, Paris, Versailles.

[12] Hattori, Y., 2001. Turbulent characteristics and transition behavior of combined-convection boundary layer along a vertical heated plate. Phd thesis, Nagoya Institute of Technology.

[13] Hattori, Y., Tsuji, T., Nagano, Y., Tanaka, N., 2000. Characteristics of turbulent combined-convection boundary layer along a vertical heated plate. International Journal of Heat and Fluid Flow 21, 520-525.

[14] Hattori, Y., Tsuji, T., Nagano, Y., Tanaka, N., 2001. Effects of freestream on turbulent combined-convection boundary layer along a vertical heated plate. International Journal of Heat and Fluid Flow 22, 315-322.

[15] Holling, M., Herwig, H., 2005. Asymptotic analysis of the near-wall region of turbulent natural convection flows. Journal of Fluid Mechanics 541, 383-397.

[16] Issa, R. I., 1985. Solution of the implicitly discretized fluid flow equations by operator-splitting. Journal of Computational Physics 62, 40-65.

[17] Issa, R. I., Gosman, A. D., Watkins, A. P., 1986. The computation of compressible and incompressible recirculating flow by a non-iterative implicit scheme. Journal of Computational Physics 62, 66-82.

[18] Jasak, H., 1996. Error Analysis and Estimation for the Finite Volume Method with Applications to Fluid Flows. Phd thesis, Imperial College, University of London.

[19] Jasak, H., 2006. Numerical Solution Algorithms for Compressible Flows. http://www.scribd.com/document/27927305/ Numerical-Solution-Algorithms-for-Compressible-Flows/.

[20] Kasagi, N., Nishimura, M., 1997. Direct numerical simulation of combined forced and natural turbulent convection in a vertical plane channel. International Journal of Heat and Fluid Flow 18, 88-99.

[21] Kitamura, K., Inagaki, T., 1987. Turbulent heat and momentum transfer of combined forced and natural convection along a vertical flat plate-aiding flow. International Journal of Heat and Mass Transfer 30, $23-41$.

[22] Krishamurthy, K., Gebhart, B., 1989. An experimental study of transition to turbulence in vertical mixed convection flows. Journal of Heat Transfer 111, 121-130.

[23] Lilly, D., 1992. A proposed modification of the germano subgrid-scale closure method. Physics of Fluids A 4, no.3, 633-635.

[24] Martin, M., Piomelli, U., Candler, G., 2000. Subgrid-scale model for compressible large-eddy simulation. Theoretical and Computational Fluid Dynamics 13, 361-376.

[25] McGrattan, K., Hostikka, S., Floyd, J., Baum, H., Rehm, R., 2005. Fire dynamics simulation (version 5) technical reference guide. Tech. rep., NIST, U.S. Department of Commerce.

[26] Moin, P., Squires, K., Cabot, W., Lee, S., 1991. A dynamic subgrid-scale model for compressible turbulence and scalar transport. Physics of Fluids A 3, no.11, 2746-2757.

[27] Nakao, K., Hattori, Y., Suto, H., 2016. Numerical investigation of a spatially developing turbulent natural convection boundary layer along a vertical heated plate. International Journal of Heat and Fluid Flow in press.

[28] Ojofeitimi, A., 2013. On the computation of buoyancy affected turbulent wall flows using large eddy simulation. Phd thesis, University of Maryland-College Park.

[29] Oliveira, P. J., Issa, R. I., 2001. An improved PISO algorithm for the computation of buoyancy-driven flows. Numerical Heat Transfer, Part B 40, 473-493.

[30] OpenCFD-Ltd., 2013. Openfoam. http://www.openfoam.com/.

[31] Patel, K., Armaly, B., Chen, T., 1998. Transition from turbulent natural convection to turbulent forced convection. Journal of Heat Transfer 120 (4), 1086-1089.

[32] Piomelli, U., Balaras, E., 2002. Wall-layer models for large-eddy simulations. Annu. Rev. Fluid Mechanics 34, 349-374.

[33] Piomelli, U., Cabot, W., Moin, P., Lee, S., 1991. Subgrid-scale backscatter in turbulent and transitional flows. Physics of Fluids A 3, 1776-1771.

[34] Piquet, J., 2003. Turbulent Flows: Models and Physics. Springer, New York, USA.

[35] To, W., Humphrey, J., 1986. Numerical simulation of buoyant,turbulent flow-I. free convection along a heated, vertical, flat plate. International Journal of Heat and Mass Transfer 29 (4), 573-592.

[36] Tsuji, T., Nagano, Y., 1988. Characteristics of a turbulent natural convection boundary layer along a vertical flat plate. International Journal of Heat and Mass Transfer 31, 1723-1734. 